$A D$

Award Number: DAMD17-02-1-0588

TITLE: Phosphorylation of hRad17 by atr is required for Cell Cycle Checkpoint Activation

PRINCIPAL INVESTIGATOR: Wei Tan

CONTRACTING ORGANIZATION: The University of Texas Health Sciences Center at San Antonio

San Antonio, TX 78229-3900

REPORT DATE: April 2005

TYPE OF REPORT: Annual Summary

PREPARED FOR: U.S. Army Medical Research and Materiel Command Fort Detrick, Maryland 21702-5012

DISTRIBUTION STATEMENT: Approved for Public Release; Distribution Unlimited

The views, opinions and/or findings contained in this report are those of the author(s) and should not be construed as an official Department of the Army position, policy or decision unless so designated by other documentation. 


\section{REPORT DOCUMENTATION PAGE}

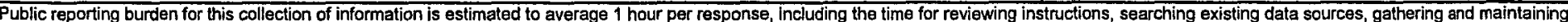

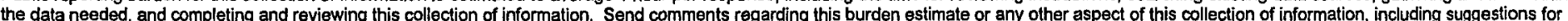

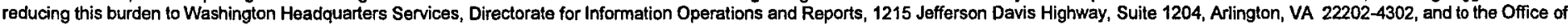
Management and Budget, Paperwork Reduction Project (0704-0188), Washington, DC 20503
1. AGENCY USE ONLY
2. REPORT DATE
April 2005

3. REPORT TYPE AND DATES COVERED

Annual Summary(1 Apr 2002 - 31 Mar 2005)

4. TITLE AND SUBTITLE

Phosphorylation of hRad17 by atr is required for Cell

5. FUNDING NUMBERS

Cycle Checkpoint Activation

DAMD17-02-1-0588

\section{AUTHOR(S)}

Wei Tan

\section{PERFORMING ORGANIZATION NAME(S) AND ADDRESS(ES)}

The University of Texas Health Sciences

Center at San Antonio

San Antonio, TX 78229-3900

E-Mail: tanweuthscsa.edu

\section{SPONSORING / MONITORING} AGENCY NAME(S) AND ADDRESS(ES)

U.S. Army Medical Research and Materiel Command

Fort Detrick, Maryland 21702-5012
8. PERFORMING ORGANIZATION REPORT NUMBER

\section{SUPPLEMENTARY NOTES}

12a. DISTRIBUTION / AVAILABILITY STATEMENT

12b. DISTRIBUTION CODE

Approved for Public Release; Distribution Unlimited

\section{ABSTRACT (Maximum 200 Words)}

Targeted protein phosphorylaytion is a key event that serves to transduce DNA damageinduced cell signals from upstream sensors to downstream effectors. ATR kinase phosphorylates BRCA1 and Rad17 upon DNA damage. As a first approach to evaluate the functional consequence of IR-induced site-specifically phosphorylated residues on BRCA1, we have established a BRCA1-dependent transcription-based assay which assesses the BRCA1-dependent repression function of ZBRK1 in mammalian cell from a reporter template bearing ZBRK1 DNA-binding sites. I found that ZBRK1 formed a homo-tetramer on the target gene promoter functioning as a DNA-binding dependent repressor as well as a DNA-binding independent corepressor. Functional dissection of ZBRK1 led to identification and characterization of a novel BRCA1-dependent repression domain encompassing ZBRKI zinc fingers 5-8 and the unique C-terminus. This C-terminal repression domain functions in a BRCA1-dependent, histone deactylase-dependent and promoter-specific manner. Significantly, BRCAl mediates ZBRK1 transcriptional repression function by modulating two properties of ZBRK1 as a transcription factor: its DNA-binding and the recruitments of other co-repressor proteins. BRCA1-dependent zBRK1 repression assay may now be exploited to evaluate the influence of IR-induced site-specific phosphorylation of BRCA1 on its sequence-specific co-repressor function.

\begin{tabular}{|l|c|c|c|}
\hline $\begin{array}{l}\text { 14. SUBJECT TERMS } \\
\text { ATR, BRCA1; Rad17, ZBRK1 and breast cancer }\end{array}$ & $\begin{array}{c}\text { 15. NUMBER OF PAGES } \\
27\end{array}$ & 16. PRICE CODE \\
\hline $\begin{array}{l}\text { 17. SECURITY CLASSIFICATION } \\
\text { OF REPORT } \\
\text { Unclassified }\end{array}$ & $\begin{array}{c}\text { 18. SECURITY CLASSIFICATION } \\
\text { OF THIS PAGE } \\
\text { Unclassified }\end{array}$ & $\begin{array}{c}\text { 19. SECURITY CLASSIFICATION } \\
\text { OF ABSTRACT } \\
\text { Unclassified }\end{array}$ & $\begin{array}{c}\text { 20. LIMITATION OF ABSTRACT } \\
\text { Unlimited }\end{array}$ \\
\hline
\end{tabular}


Table of Contents

Cover. 1

SF 298. 2

Table of Contents .3

Introduction. .4

Body.. ..4-6

Key Research Accomplishments..........................................6

Reportable Outcomes..........................................................6

Conclusions......................................................................6-7

References............................................................................

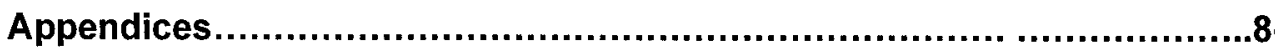




\section{INTRODUCTION}

Targeted protein phosphorylation is a primary cellular response to DNA damage and a signal event through which downstream effector molecules are mobilized into action by upstream DNA damage response sensors. BRCA1 (Breast Cancer 1), a hereditary breast- and ovarian-specific tumor suppressor, plays a central role in the cellular response to DNA damage by virtue of its dual function in DNA damage-induced cell cycle checkpoint control and DNA repair. In response to DNA damage, BRCA1 can be phosphorylated by both the ATM (ㅅtaxia telangiecstasia mutated) and ATR ( ATM

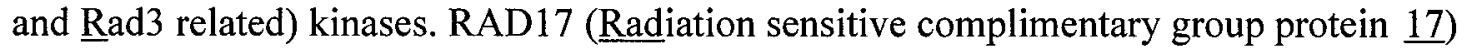
is another ATR substrate and downstream DNA damage-respone effector molecule. At the molecular level, Rad17 is phosphorylated, and this phosphorylation is required for DNA damage-induced cell cycle checkpoint control during G1 phase in breast cancer cells. Over-expression of human RAD17 (hRAD17) has been linked to metastastic breast cancer. A better understanding of how DNA damage-induced BRCA1 and hRAD17 phosphorylation modulate their respective functions may reveal novel insight into the molecular events underlying breast tumorigeneis and metastasis.

\section{BODY}

In an initial effort to investigate the influence of phosphorylation on BRCA1, I have established a BRCA1-dependent transcription-based assay to evaluate the effects of IRinduced site-specifically phosphorylated residues on BRCA1. This assay is based on the functional interaction between BRCA1 and the sequence-specific transcriptional repressor protein ZBRK1 (Zinc Finger BRCA1 interacting protein with a KRAB domain 1). Previously, our lab showed that BRCA1 is a co-repressor of ZBRK1, a sequencespecific DNA-binding transcriptional repressor of the DNA damage-inducible GADD45 gene that functions in $\mathrm{G} 2 / \mathrm{M}$ cell cycle checkpoint control (1). In addition to GADD45,

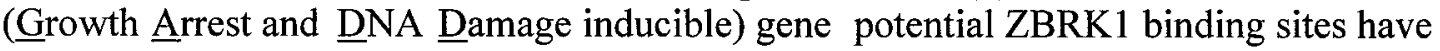
been identified in other DNA damage-inducible genes, indicating a prospective global role for ZBRK1 and BRCA1 in the coordinate regulation of DNA damage-response genes (1). Based on these previous observations, we have proposed a model whereby ZBRK1 and BRCA1 coordinately repress a group of DNA damage response genes in the absence of genotoxic stress and, further, that DNA damage-induced cell signals relieve this repression, thereby permitting DNA damage-induced activation of these genes.

The DNA damage-induced cell signals that relieve coordinate repression of DNA damage response genes by ZBRK 1 and BRCA1 is likely to involve phosphorylation. In fact, previous studies have revealed that IR-induced protein phosphorylation is required to relieve BRCA1-mediated repression of the GADD45 (2). To facilitate studies designed to determine the effects of targeted mutations at identified sites of IR (ionizing radiation-induced) phosphorylation within BRCA1 on its ability to control transcription, we established a BRCA1-dependent ZBRK1 transcriptional repression assay. This assay is designed to assess the BRCA1-dependent repression function of ZBRK1 in mammalian cells from a reporter template bearing ZBRK1 DNA-binding sites. To establish the utility of this system as a means to study BRCA1-dependent ZBRK1 
repression, we used this system as a functional readout during experiments designed to functionally dissect ZBRK1. This study was completed and published within this training period (3). In this study, we reported the identification and characterization of a novel BRCA1-dependent transcriptional repression domain within ZBRK1 composed of ZBRK1 zinc fingers 5-8 along with sequences in the unique ZBRK1 C-terminus. This Cterminal repression domain functions in a $\mathrm{BRCA1} 1-$, histone deacetylase-, and promoterspecific manner and is thus functionally distinguishable from an $\mathrm{N}$-terminal repression domain in ZBRK1, which exhibits no BRCA1 dependency and broad promoter specificity. Significantly, we also found that the BRCA1-dpendent transcriptional repression domain on ZBRK1 includes elements that modulate its sequence-specific DNA-binding activity. In addition to demonstrating the utility of this assay to quantify BRCA1-dependent ZBRK1 transcriptional repression, this study also revealed an unanticipated dual function for the ZBRK1 zinc fingers in DNA-binding and BRCA1dpendent transcriptional repression. When ZBRK1 KRAB repression domain was replaced by VP16 transactivation domain, the chimera can transactivate transcription controlled by ZBRK1 DNA binding sites only in presence of BRCA1 and ZBRK1 mutations which disrupted ZBRK1-BRCA1 interaction compromised BRCA1 dependent transactivation of the chimera. This evidence indicates that BRCA1 is required for ZBRK1 to be targeted to its DNA binding site in vivo. As such, this study sheds new light on the mechanistic basis by which BRCA1 mediates sequence-specific control of DNA damage-responsive gene transcription.

ZBRK1 is a member of KRAB-zinc finger protein family which consists of about 300 transcriptional repressors. Biochemical analyses, including protein cross-linking and gel filtration chromatography, revealed that ZBRK1 homo-oligomers existed as tetramers in solution. Functionally, ZBRK1 oligomerization supports ZBRK1-directed transcriptional repression through ZBRK1 response elements (ZREs); structural requirements for oligomerization-dependent repression include the CTRD and the KRAB repression domains within ZBRK1. This observation suggests that higher order homooligomers of ZBRK1 may assemble on target ZREs through both protein-DNA and CTRD-dependent protein-protein interactions. This part of my work which extends current understanding of the functional mode of ZBRK1 has also been published in this training period (4).

Our BRCA1-dependent ZBRK1 repression assay may now be exploited to evaluate the influence of IR-induced site-specific phosphorylation of BRCA1 on its sequence-specific co-repressor function. We plan to evaluate the influence of IR on the ability of ectopically expressed wild-type BRCA1 to function as a ZBRK1-specific corepressor. We predict, and will test the possibility, that IR leads to phosphorylation of BRCA1 and consequent relief of ZBRK1-directed repression from the ZBRK1 target reporter plasmid through disruption of the ZBRK1/BRCA1 interaction or conversion of BRCA1 from a corepressor to a coactivator. Subsequently, we will evaluate BRCA1 derivatives bearing site-directed mutations at sites of IR-induced phosphorylation for their respective abilities to function as IR-reversible co-repressors of ZBRK1. We predict that BRCA1 derivatives bearing mutations at identified sites of IR-induced phosphorylation will function as constitutive ZBRK1 co-repressors through an IRinsensitive interaction with ZBRK1. This assay should therefore provide us with a rapid 
and facile approach to test the hypothesis that IR-induced site-specific phosphorylation of BRCA1 modulates its transcriptional regulatory activities.

\section{KEY RESEARCH ACCOMPLISHMENTS TO DATE}

- Novel discovery within ZBRK1 of functionally bipartite zinc fingers with dual roles in sequence-specific DNA-binding and BRCA1-dependent transcriptional repression.

- Establishment of a BRCA1-dependent ZBRK1 transcriptional repression assay that will expedite studies designed to test the hypothesis that IR-induced sitespecific phosphorylation of BRCAl modulates its transcriptional regulatory activities.

- Reveal of the mechanistic mode in which ZBRK1 function as both a DNAbinding dependent transcriptional repressor as well as a DNA-binding independent corepressor.

\section{REPORTABLE OUTCOMES TO DATE.}

\section{Manuscripts:}

1. Tan, W., Kim, S. and Boyer T.G. (2004). Tetrameric oligomerization mediates transcriptional repression by the BRCAl-dependent KRAB-zinc finger protein ZBRK1. J Biol. Chem. 279(53):55153-55160.

2. Tan, W. Zheng, L., Lee, W.-H. and Boyer, T.G. (2004). Functional dissection of Transcription Factor ZBRK1 Reveals Zinc Fingers with Dual Roles in DNAbinding and BRCA1-dependent Transcriptional Repression. J. Biol. Chem. 279: 6576-6587.

\section{Meeting Abstract:}

1. Tan, W. and Boyer, T.G. (2004). BRCA1 modulates the transcriptional repression of ZBRK1 through targeting its DNA binding and post-DNA binding repression function. The $13^{\text {th }}$ Annual Cancer Research Symposium. San Antonio, TX.

2. Tan, W. Zheng, L., Chen, P.-L., Lee, W.-H., and Boyer, T.G. (2003). Molecular dissection of a BRCA1-dependent transcriptional repression domain. Mechanisms of Eukaryotic Transcription. Cold Spring Harbor, New York.

\section{CONCLUSIONS}

We have succeeded establishing an efficient and reliable BRCA1-dependent transcription-based functional assay which has permitted us to make the additional novel discovery that ZBRK1, a BRCA1-dependent transcriptional repressor, harbors zinc 
fingers with dual roles in sequence-specific DNA-binding and BRCA1-dependent transcriptional repression. Additionally, ZBRK1 forms homo-tetramer on its target gene promoter functioning in both DNA-binding dependent and independent manner. This finding sheds new light on the mechanistic basis by which BRCA1 mediates sequencespecific control of DNA damage-responsive gene transcription. Our current efforts are targeted at identifying DNA damage-induced site-specific phosphorylation events with potential functional relevance to the role of BRCA1 in transcription. Hence, we will exploit these BRCA1-dependent transcription-based assays to analyze of the effects of targeted BRCA1 mutations at identified sites of phosphorylation on its transcriptional regulatory activities. These studies should illuminate further the molecular basis for the caretaker properties of BRCA1.

\section{REFERENCES}

1. Zheng, L., Pan, H., Li, S., Fleskin-Nitikin, A., Chen, P.-L., Boyer, T.G., and Lee, W.H. (2000). Sequence-Specific Transcriptional Corepressor Function for BRCA1 through a Novel Zinc Finger Protein, ZBRK1. Mol. Cell 6: 757-768.

2. Li, S., Ting, N.S.Y., Zheng, L., Chen, P.-L., Ziv, Y., Shiloh, Y., Lee, E.Y.-H., and Lee, W.-H. (2000). Functional link of BRCAl and ataxia telangiectasia gene product in DNA damage response. Nature 406, 210-215.

3. Tan, W., Zheng, L., Lee, W.-H. and Boyer, T.G. (2004). Functional dissection of Transcription Factor ZBRK1 Reveals Zinc Fingers with Dual Roles in DNA-binding and BRCA1-dependent Transcriptional Repression. J. Biol. Chem. 279: 6576-6587.

4. Tan, W., Kim, S. and Boyer T.G. (2004). Tetrameric oligomerization mediates transcriptional repression by the BRCA1-dependent KRAB-zinc finger protein ZBRK1. J Biol. Chem. 279(53):55153-55160. 


\title{
Functional Dissection of Transcription Factor ZBRK1 Reveals Zinc Fingers with Dual Roles in DNA-binding and BRCA1-dependent Transcriptional Repression*
}

Received for publication, November 10, 2003, and in revised form, November 26, 2003 Published, JBC Papers in Press, December 2, 2003, DOI 10.1074/jbc.M312270200

\author{
Wei Tanł, Lei Zheng\$, Wen-Hwa Lee§, and Thomas G. Boyer $\neq$ \\ From the $\ddagger$ Department of Molecular Medicine and Institute of Biotechnology, University of Texas Health Science Center, \\ San Antonio, Texas 78245-3207 and the \$Department of Biological Chemistry, University of California, \\ Irvine, California 92697-1700
}

The breast- and ovarian-specific tumor suppressor BRCA1 has been implicated in both activation and repression of gene transcription by virtue of its direct interaction with sequence-specific DNA-binding transcription factors. However, the mechanistic basis by which BRCA1 mediates the transcriptional activity of these regulatory proteins remains largely unknown. To clarify this issue, we have examined the functional interaction between BRCA1 and ZBRK1, a BRCA1-dependent KRAB eight zinc finger transcriptional repressor. We report here the identification and molecular characterization of a portable BRCA1-dependent transcriptional repression domain within ZBRK1 composed of zinc fingers 5-8 along with sequences in the unique ZBRK1 $\mathrm{C}$ terminus. This $\mathrm{C}$-terminal repression domain functions in a BRCA1-, histone deacetylase-, and promoter-specific manner and is thus functionally distinguishable from the $\mathrm{N}$-terminal $\mathrm{KRAB}$ repression domain in ZBRK1, which exhibits no BRCA1 dependence and broad promoter specificity. Significantly, we also find that the BRCA1-dependent transcriptional repression domain on ZBRK1 includes elements that modulate its sequence-specific DNA binding activity. These findings thus reveal the presence within ZBRK1 of functionally bipartite zinc fingers with dual roles in sequence-specific DNA-binding and BRCA1-dependent transcriptional repression. We discuss the implications of these findings for the role of BRCA1 as ZBRK1 co-repressor.

Germ line inactivation of the gene encoding BRCA1 confers a cumulative lifetime risk of female breast and ovarian cancer (1-3). Although the mechanistic basis for its tissue- and gender-specific tumor suppressor activity remains poorly defined, BRCA1 nonetheless fulfills a broad function in the maintenance of global genome stability (4-10). The underlying basis for this caretaker activity likely derives from the role of BRCA1 as a conduit in the cellular DNA damage response, wherein it serves to couple DNA damage-induced signals to downstream

* This work was supported by United States Army Department of Defense Grant DAMD17-02-1-0584 (to T. G. B.), United States Army Department of Defense Training Grant (to W. T.), and National Institutes of Health Grants CA94170, CA81020 (to W.-H. L.), and CA098301-01 (to T. G. B). The costs of publication of this article were defrayed in part by the payment of page charges. This article must therefore be hereby marked "advertisement" in accordance with 18 U.S.C. Section 1734 solely to indicate this fact.

I To whom correspondence should be addressed: Dept. of Molecular Medicine and Institute of Biotechnology, University of Texas Health Science Center, 15355 Lambda Dr., San Antonio, TX 78245-3207. Tel. 210-567-7258; Fax: 210-567-7377; E-mail: boyer@uthsesa.edu. responses including DNA damage repair and cell cycle checkpoint activation $(6,7,11-21)$. Several potentially overlapping cellular activities have been ascribed to BRCA1, each of which could underlie its ability to control signal output. For example, BRCA1 has been implicated in chromatin remodeling, ubiquitinylation, recombination, and transcriptional regulation $(6,22$ 28 ). The extent to which these pleiotropic activities contribute to the caretaker function of BRCA1 is presently unknown; however, the fact that each of these BRCA1-associated activities are similarly abrogated by cancer-predisposing BRCA1 missense mutations suggests a strong correlative link between their discharge and BRCA1-mediated tumor suppression.

With respect to its role in transcription control, BRCA1 has been implicated in both activation and repression of genes linked to a variety of biological processes, including cell growth control and DNA replication and repair $(21,29-31)$. Thus, by virtue of its transcriptional regulatory activity, BRCA1 could influence cellular responses downstream of DNA damage signals, and this activity could contribute to its caretaker function.

The precise role of BRCA1 in gene-specific transcription control has yet to be definitively established. Because it exhibits no sequence-specific DNA binding activity, it seems likely that BRCA1 is targeted to specific genes through its functional interaction with sequence-specific DNA-binding transcription factors. Direct evidence to support this hypothesis has come from the identification of multiple DNA-binding transcription factors with which BRCA1 has been shown to physically interact and functionally synergize, including p53, c-Myc, estrogen receptor $\alpha$, androgen receptor, OCT-1, NF-YA, and ZBRK1 $(32-40)$. However, the underlying mechanism by which BRCA1 mediates the transcriptional stimulatory or repressive effects of these regulatory proteins has not been established.

We have been studying the functional interaction between BRCA1 and the transcriptional repressor ZBRK1 as a model system to understand the mechanistic basis by which BRCA1 mediates sequence-specific transcription control. Initially identified by virtue of its physical interaction with BRCA1, ZBRK1 (Zinc finger and BRCA1-interacting protein with a KRAB domain 1 ) is a member of the Kruppel-associated box-zinc finger protein (KRAB-ZFP) family of transcriptional repressors (39, 41). Typically, KRAB-ZFPs bind to their corresponding target genes through tandem $\mathrm{C}$-terminal $\mathrm{C}_{2} \mathrm{H}_{2}$ zinc fingers and repress transcription through an $\mathrm{N}$-terminal $\mathrm{KRAB}$ domain, which silences gene expression through the indirect recruitment of histone deacetylases, histone methyltransferases, and heterochromatin proteins (41-47). Like other KRAB-ZFPs, ZBRK1 harbors an N-terminal KRAB domain. However, ZBRK1 is atypical among KRAB-ZFPs due to the fact that it harbors 8 central $\mathrm{C}_{2} \mathrm{H}_{2}$ zinc fingers and a unique $\mathrm{C}$ terminus 
that is absent among the larger family of KRAB-ZFPs.

Through its 8 central zinc fingers, ZBRK1 binds to a compositionally flexible 15-bp DNA sequence, GGG $x x x$ CAG $x x x$ TTT (where $x$ is any nucleotide) (39). A search for potential ZBRK1 DNA-binding sites in existing genes led to intron 3 of GADD45a, a functionally important DNA damage-response effector known to be regulated transcriptionally by BRCA1 (21, 32, 39). Functional analysis revealed that ZBRK1 represses $G A D D 45 a$ gene transcription through its intron 3 DNA-binding site in a BRCA1-dependent manner, thus revealing BRCA1 to be a ZBRK1 co-repressor (39). Significantly, familial breast cancer-derived mutants of BRCA1 that disrupt its interaction with ZBRK1 abrogate its co-repressor activity, suggesting that its co-repressor function may be important for the tumor suppressor properties of BRCA1 (39). The regulation of GADD45a gene transcription is likely to be complex and controlled coordinately by ZBRK1 and BRCA1 in concert with other transacting factors, including p53, OCT1, and NF-YA, that function through cis-acting sequences present in the GADD45a promoter and intron 3 regions $(21,32,39,48,49)$.

In addition to $G A D D 45 a$, potential ZBRK1-binding sites have been identified in other DNA damage-response genes that are also regulated by BRCA1, including $\mathrm{p} 21, \mathrm{Bax}$, and GADD153 (39). This observation suggests a potentially broader role for BRCA1 and ZBRK1 in the coordinate transcriptional regulation of diverse DNA damage-response genes. To begin to explore the mechanism by which BRCA1 mediates sequencespecific transcriptional repression through ZBRK1, we have pursued in greater depth the physical and functional interaction between these two proteins. We report here the identification and molecular characterization of a portable BRCA1-dependent transcriptional repression domain within ZBRK1 composed of zinc fingers 5-8 along with sequences in the unique $\mathrm{ZBRK} 1 \mathrm{C}$ terminus. This $\mathrm{C}$-terminal repression domain functions in a BRCA1-, histone deacetylase (HDAC)-, ${ }^{1}$ and promoter-specific manner and is thus functionally distinguishable from the N-terminal KRAB repression domain in ZBRK1, which exhibits no BRCA1 dependence and broad promoter specificity. Significantly, we also find that the BRCA1-dependent C-terminal transcriptional repression domain within ZBRK1 is composed of elements that modulate sequence-specific DNA-binding by zinc fingers 1-4. These findings thus reveal an unanticipated dual function for the ZBRK1 zinc fingers in DNA binding and transcriptional repression, and further shed new light on the mechanistic role of BRCA1 in sequence-specific transcriptional control.

\section{EXPERIMENTAL PROCEDURES}

\section{Plasmid Construction and Mutagenesis}

Expression Plasmids-pMAL-C2-TEV-ZBRK1 $\Delta \mathrm{K}$ for expressing MBP-ZBRK1 $\triangle \mathrm{K}$ in Escherichia coli was derived from pGEPK3-ZBRK1 $\Delta \mathrm{K}$, which is a derivative of pCNF-ZBRK1 $\Delta \mathrm{K}$ (39). Briefly, a BamHIXhoI fragment carrying ZBRK1 cDNA sequences encoding ZBRK1 amino acids 144-532 (lacking the $\mathrm{N}$-terminal KRAB domain) was subcloned into the BamHI and XhoI sites of pMAL-C2-TEV (provided by Dr. P. Renee Yew), thereby generating a translational fusion of MBP and ZBRK1 $\Delta \mathrm{K}$. C-terminal truncation mutants of MBP-ZBRK1 $\Delta \mathrm{K}$ bearing stepwise deletions of individual ZBRK1 zinc fingers were expressed from pMAL-C2-TEV-ZBRK1 $\triangle \mathrm{K}$ deletion derivatives, each of which was constructed by PCR-based subcloning. Briefly, sequences encoding ZBRK1 $\Delta \mathrm{K}$ within pMAL-C2-TEV-ZBRK1 $\Delta \mathrm{K}$ were liberated as a BamHI-HindIII fragment and replaced with corresponding PCR-

\footnotetext{
${ }^{1}$ The abbreviations used are: HDAC, histone deacetylase; EMSA, electrophoretic mobility shift assay; MEFs, mouse embryo fibroblasts; aa, amino acids; MBP, maltose-binding protein; PMSF, phenylmethylsulfonyl fluoride; TSA, trichostatin A; ZRE, ZBRK1-response element; $\mathrm{BF}$, broken finger; TK, thymidine kinase; WT, wild type; Mut., mutant; HSV, herpes simplex virus.
}

generated ZBRK1 deletion fragments using a common upstream primer corresponding to MBP sequences and unique downstream primers within the $\mathrm{C}$ terminus of each zinc finger (defined here as the seventh amino acid residue C-terminal to the last histidine residue of each $\mathrm{C}_{2} \mathrm{H}_{2}$ zinc finger). Individual MBP-ZBRK $1 \triangle \mathrm{K}$ deletion derivatives encode the following ZBRK1 amino acids (aa): MBP-ZBRK1 $\triangle \mathrm{K}$ 8ZF (aa 144-431); 7ZF (aa 144-403); 6ZF (aa 144-377); 5ZF (aa 144-347); 4ZF (aa 144319); $3 Z F$ (aa 144-291); 2ZF (aa 144-263); and 1ZF (aa 144-235). Individual MBP-ZBRK1 $\triangle \mathrm{K}$ broken finger (BF) mutants were generated by PCR-based site-directed mutagenesis of pMAL-C2-TEV-ZBRK1 $\Delta \mathrm{K}$ using the QuickChange II site-directed mutagenesis kit following the manufacturer's recommendations (Stratagene, La Jolla, CA). Each broken finger mutant bears a histidine (CAT codon) to asparagine (aaT codon) substitution mutation at the first of the two conserved histidine residues within the targeted $\mathrm{C}_{2} \mathrm{H}_{2}$ zinc finger.

ZBRK1 5ZFC was expressed as a GAL4 DNA-binding domain fusion in mammalian cells from the plasmid GAL4-ZBRK1 5ZFC, constructed by subcloning a PCR-amplified ZBRK1 cDNA fragment encoding amino acids 319-532 (encompassing zinc finger 5 through the $C$ terminus) into the SalI and HindIII sites of pM (Clontech, Palo Alto, CA). N- and C-terminal truncation derivatives of GAL4-ZBRK1 5ZFC bearing stepwise deletions of individual zinc fingers and C-terminal sequences, respectively, were generated by PCR-based subcloning. Briefly, the SalI-HindIII fragment within GAL4-ZBRK1 5ZFC encoding ZBRK1 5ZFC was replaced with corresponding PCR-generated ZBRK1 deletion fragments. Individual GAL4-ZBRK1 5ZFC deletion derivatives encode the following ZBRK1 amino acids (aa): GAL4-ZBRK1 6ZFC (aa 347532); 7ZFC (aa 375-532); 8ZFC (aa 403-532); C (aa 431-532); GAL4ZBRK1 5ZFC $\Delta 1$ (aa 319-523); 5ZFC $\Delta 2$ (aa 319-503); and 5ZFC $\Delta 3$ (aa 319-483). Broken finger derivatives of GAL4-ZBRK1 5ZFC were generated by PCR amplification of ZBRK1 sequences encoding amino acids 319-532 from individual pMAL-C2-TEV ZBRK1 $\triangle \mathrm{K} B F$ mutants and subsequent replacement of the SalI-HindIII wild-type ZBRK1 fragment in GAL4-ZBRK1 5ZFC.

ZBRK1 5ZFC and its truncation and broken finger derivatives were expressed in yeast as GAL4 activation domain fusions using pGADT7 (Clontech, Palo Alto, CA). Briefly, ZBRK1 5ZFC and its truncation (6ZFC, 7ZFC, 8ZFC, 0ZFC, 5ZFC $\Delta 1,5 Z$ FC $\Delta 2$, and 5ZFC $\Delta 3$ ) and broken finger (BF5, BF6, BF7, and BF8) derivatives were excised as BamHI-blunted SalI fragments from GAL4-ZBRK1 5ZFC and its corresponding derivative plasmids, and subcloned into the BamHI and blunted XhoI sites in pGADT7. A BRCA1 cDNA fragment (encoding amino acids 341-748) encompassing the ZBRK1-binding domain (39) was PCR-amplified and subcloned into the EcoRI and SalI sites of pGBKT7, thereby generating a translational fusion of the GAL4 DNAbinding domain with BRCA1 amino acids $341-748$ in the plasmid pGBKT7-BRCA1.

The ZBRK1 KRAB domain was expressed as a GAL4 DNA-binding domain fusion in mammalian cells from GAL4-ZBRK1 KRAB, constructed by subcloning a PCR-amplified ZBRK1 cDNA fragment encoding amino acids 1-85 into the BamHI and blunted XbaI sites of $\mathrm{pM} 2$ (50). All PCR-based subcloning was performed using Pfu DNA polymerase (Stratagene, La Jolla, CA), and the integrity of individual deletion and substitution mutations was confirmed by DNA sequence analysis.

Reporter Plasmids- $\mathrm{pG}_{5}$ TK-Luc carrying five copies of the GAL4 DNA-binding site upstream of the herpes simplex virus thymidine kinase (TK) promoter (sequences corresponding to -105 to +51 , where +1 is the transcription start site) driving expression of the gene encoding firefly luciferase was constructed by replacing a HindIII-BglII fragment from pSBS-GAL-TK-Luc (provided by Dr. Tony Ip) with a HindIIIBglII fragment from $\mathrm{pG}_{5}$ TK-CAT (provided by Dr. P. Renee Yew), thus positioning five copies of the GAL4 DNA-binding site and the TK promoter upstream of the firefly luciferase gene. $\mathrm{pG}_{5} \mathrm{SV} 40$-Luc carrying five GAL4 DNA-binding sites upstream of the SV40 promoter driving expression of the firefly luciferase gene has been described previously (39). pG $_{5}$ SNRPN-Luc (provided by Dr. Paul A. Wade) carries five GAL4 DNA-binding sites upstream of the human small nuclear ribonucleoprotein $\mathrm{N}$ promoter driving expression of the firefly luciferase gene (51).

\section{Recombinant Protein Expression and Purification}

MBP-ZBRK1 fusion proteins were expressed in and purified from $E$. coli strain BL21 Star (DE3) pLysS (Invitrogen). Briefly, cells were grown at $37^{\circ} \mathrm{C}$ to an $A_{600}$ of 0.6 . Isopropyl-1-thio- $\beta$-D-galactopyranoside was added to a final concentration $0.3 \mathrm{~mm}$, and the cells were transferred to $25^{\circ} \mathrm{C}$ for another $3.5 \mathrm{~h}$. Cells were pelleted, washed once with phosphate-buffered saline, and then resuspended in MBP binding buffer (25 mM Tris-HCl, pH 7.5; 1 mM EDTA; $200 \mathrm{~mm} \mathrm{NaCl;} 20 \mu \mathrm{M}$ 
A

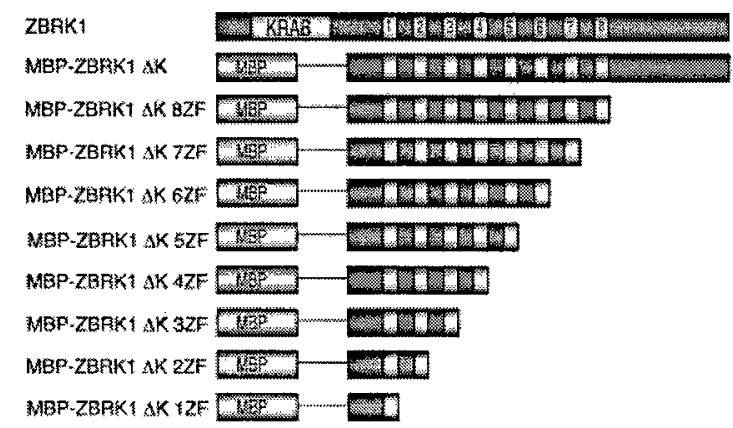

B

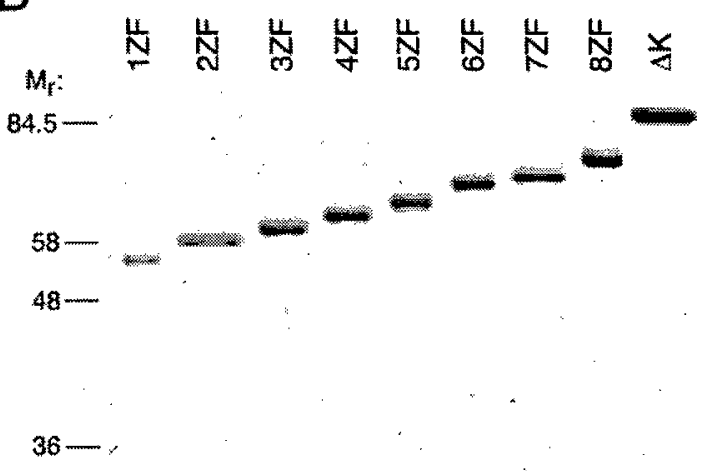

C

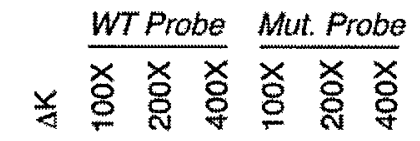

D

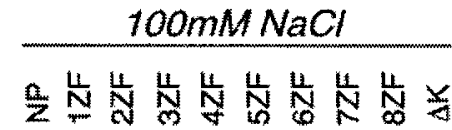

$E$

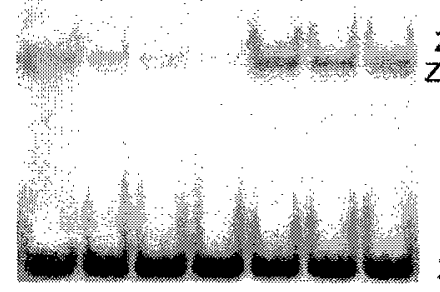

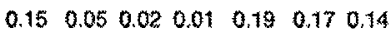

ZREZBRK1

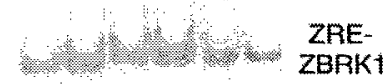

ZRE

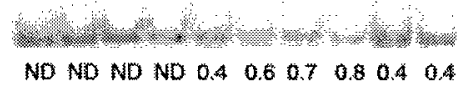

ZRE

ND ND ND ND $0.26 \quad 0.650 .72 \quad 0.190 .22 \mathrm{ND}$

FTG. 1. Identification of DNA binding determinants on ZBRK1. $A$, schematic representation of ZBRK1 (the KRAB domain and numbered zinc fingers are indicated), MBP-ZBRK1 $\triangle \mathrm{K}$, and MBP-ZBRK1 $\triangle \mathrm{K}$ truncation derivatives. $B$, purified MBP-ZBRK1 $\triangle \mathrm{K}$ and its corresponding truncation derivatives as indicated were resolved by SDS-10\% PAGE and visualized by Comassie Blue staining. Molecular weight marker positions $\left(M_{r}\right)$ are indicated. $C$, competition EMSA. EMSA was performed using a ${ }^{32}$ P-labeled double-stranded oligonucleotide probe corresponding to a wild-type consensus ZRE and purified MBP-ZBRK1 $\triangle \mathrm{K}$ (1st lane). A 100-, 200-, or 400-fold molar excess of an unlabeled wild-type (WT) ZRE probe (2nd to 4th lanes) or mutated (Mut.) ZRE probe corresponding to a double-stranded oligonucleotide identical in length but different in sequence (5th to 7 th lanes) was added to the binding reaction as indicated. The positions of the unbound ZRE oligonucleotide probe (ZRE) and the ZRE-MBP-ZBRK1 $\triangle \mathrm{K}$ nucleoprotein complex (ZRE-ZBRK1) are indicated. For each EMSA reaction, the proportional fraction of ZRE probe bound by MBP-ZBRK1 $\Delta K$ is indicated below each lane and was determined by dividing the number of radioactive counts in the bound probe by the number of radioactive counts in the bound plus the unbound probe. $D$ and $E$, sequence-specific DNA binding activity of MBP-ZBRK1 $\triangle \mathrm{K}$ and its corresponding truncation derivatives. EMSA was performed using the consensus ZRE probe and $50 \mathrm{ng}$ of either MBP-ZBRK1 $\triangle \mathrm{K}$ or each of its corresponding deletion derivatives as indicated. $N P$ indicates no protein added to the EMSA reaction. The positions of the unbound ZRE oligonucleotide probe $(Z R E)$ and the ZRE-MBP-ZBRK1 $\triangle \mathrm{K}$ nucleoprotein complex (ZRE-ZBRK1) are indicated. DNA-binding reactions were performed in $100 \mathrm{mM} \mathrm{NaCl}(D)$ or $200 \mathrm{mM} \mathrm{NaCl}(E)$. For each EMSA reaction, the proportional fraction of ZRE probe bound by MBP-ZBRK1 $\triangle \mathrm{KK}$ or its truncation derivatives is indicated below each lane and was determined by dividing the number of radioactive counts in the bound probe by the number of radioactive counts in the bound plus the unbound probe. $N D$, not detectable.

$\mathrm{ZnCl}_{2}$; and $10 \mathrm{mM} \beta$-mercaptoethanol) supplemented with protease inhibitors (aprotinin $0.4 \mu \mathrm{g} / \mathrm{ml}$; chymostatin $0.5 \mu \mathrm{g} / \mathrm{ml}$; leupeptin 0.5 $\mu \mathrm{g} / \mathrm{ml}$; pepstatin $0.5 \mu \mathrm{g} / \mathrm{ml}$; PMSF $0.5 \mathrm{mM}$; and benzamidine- $\mathrm{HCl} 0.5$ $\mathrm{mM})$. Resuspended cells were frozen and thawed one time, followed by sonication ( 3 times for $1 \mathrm{~min}$ ) and clarification by centrifugation at $30,000 \times g$ for $30 \mathrm{~min}$. MBP-ZBRK1 fusion proteins were purified from clarified lysates by affinity chromatography on amylose resin (New England Biolabs, Beverly, MA). Briefly, clarified lysates were incubated with amylose resin in batch for $1 \mathrm{~h}$ at $4{ }^{\circ} \mathrm{C}$, washed 3 times with $\mathrm{MBP}$ binding buffer, and then eluted with MBP binding buffer containing $0.5 \%$ maltose for $30 \mathrm{~min}$ at $4^{\circ} \mathrm{C}$. Purified proteins (estimated to be $>95 \%$ homogeneous by SDS-PAGE and subsequent visualization by Coomassie Blue staining) were dialyzed for $1 \mathrm{~h}$ at $4{ }^{\circ} \mathrm{C}$ against EMSA storage buffer (25 mM Tris-HCl, pH 7.5; $100 \mathrm{mM} \mathrm{NaCl} ; 20 \mu \mathrm{M} \mathrm{ZnCl} l_{2} ; 10 \%$ glycerol; $10 \mathrm{mM} \beta$-mercaptoethanol; and $0.5 \mathrm{mM}$ PMSF) before long term storage at $-80^{\circ} \mathrm{C}$.

\section{Electrophretic Mobility Shift Assay (EMSA)}

For EMSA, the ZRE probe was obtained by annealing two complementary oligonucleotides corresponding in sequence to the consensus ZBRK1 DNA-binding site: 5'-GATCCACGGGACGCAGGTGTTTTGTGCCG-3' and 5'-GATCCGGCACAAAACACCTGCGTCCCGTG-3' (39). Mutant (Mut) probe was obtained by annealing two oligonucleotides,
5'-GATCCACCTCACGTTCGTGCACTGTGCCG-3' and 5'-GATCCGGCACAGTGCACGAACGTGAGGTG-3' (39). Each of these doublestranded probes carried overhanging ends, which were filled in with $\left[\alpha^{32} \mathrm{P}\right] \mathrm{dCTP}$ by Klenow enzyme. In each reaction, purified MBP-ZBRK1 fusion proteins (50 $\mathrm{ng}$ ) were incubated with $6000 \mathrm{cpm}$ of a ${ }^{32} \mathrm{P}$-labeled double-stranded oligonucleotide probe in $30 \mu \mathrm{l}$ of EMSA binding buffer (25 mM Tris-HCl pH 7.5; $20 \mu \mathrm{MnCl}_{2} ; 12.5 \%$ glycerol; $0.5 \mathrm{mM}$ PMSF; and a variable concentration of $\mathrm{NaCl}$ as indicated). Following $30 \mathrm{~min}$ of incubation at room temperature, reaction mixtures were loaded onto a $5 \%$ non-denaturing polyacrylamide gel and electrophoresed at $200 \mathrm{~V}$ for $2 \mathrm{~h}$ at $4^{\circ} \mathrm{C}$ in $0.5 \times \mathrm{TBE}$. Dried gels were subjected to PhosphorImager analysis (Amersham Biosciences).

\section{Cell Culture, Transfections, and Reporter Assays}

Brca1-/-, p53-/- (Brca1-/-), and p53-/- (Brca1+/+) mouse embryo fibroblasts (MEFs) (39) and U2OS human osteosarcoma cells were cultured in Dulbecco's modified Eagle's medium (Invitrogen) supplemented, with $10 \%$ fetal bovine serum (Hyclone, Logan, UT). Brca1+l+, Brca1-1-, and U2OS cells were transfected at $60 \%$ confluency using Effectene reagent (Qiagen, Valencia, CA), and the expression and reporter plasmids are indicated in each figure. Each transfection also included an internal control plasmid, pCH110 (40), expressing $\beta$-galactosidase under control of the SV40 promoter. Forty-eight hours 
FIG. 2. Identification of DNA-binding determinants on ZBRK1. $A$, schematic representation of ZBRK1, MBPZBRK1 $\Delta \mathrm{K}$, and MBP-ZBRK1 $\Delta \mathrm{K}$ broken finger derivatives. $B$, purified MBPZBRK1 $\Delta \mathrm{K}$ and its corresponding broken finger derivatives as indicated were resolved by SDS-10\% PAGE and visualized by Coomassie Blue staining. Molecular weight marker positions $\left(M_{\mathrm{r}}\right)$ are indicated. $C$, sequence-specific DNA binding activity of MBP-ZBRK $1 \triangle \mathrm{K}$ and its corresponding broken finger derivatives. EMSA was performed using the consensus ZRE probe and $50 \mathrm{ng}$ of either MBPZBRK1 $\triangle \mathrm{K}$ or each of its corresponding broken finger derivatives as indicated. $N P$ indicates no protein added to the EMSA reaction. The positions of the unbound ZRE oligonucleotide probe (ZRE) and the ZRE'MBP-ZBRK1 $\Delta \mathrm{K}$ nucleoprotein complex (ZRE-ZBRK1) are indicated. DNA-binding reactions were performed in $200 \mathrm{~mm} \mathrm{NaCl}$. For each EMSA reaction, the proportional fraction of ZRE probe bound by MBP-ZBRK1 $\Delta \mathrm{K}$ or its broken finger derivatives is indicated below each lane and was determined by dividing the number of radioactive counts in the bound probe by the number of radioactive counts in the bound plus the unbound probe. $N D$, not detectable.
A

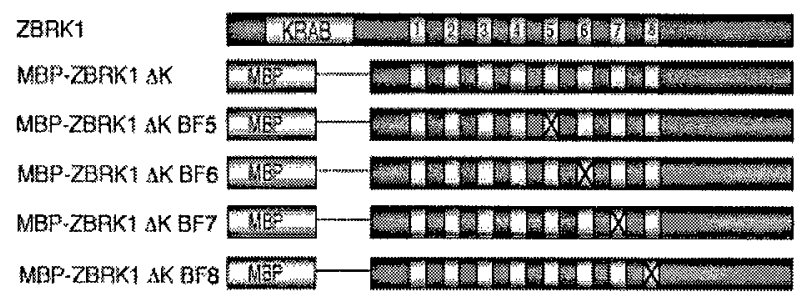

B

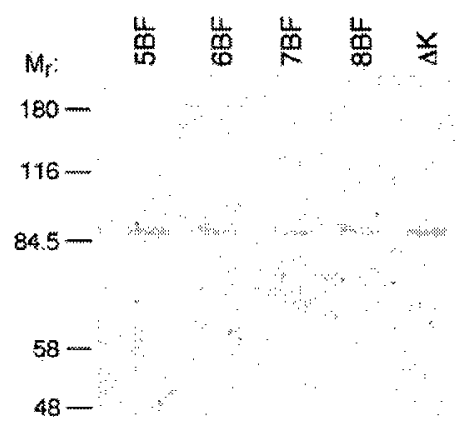

C

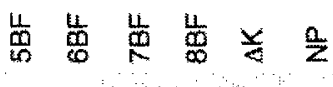

ZRE. ZBRK1

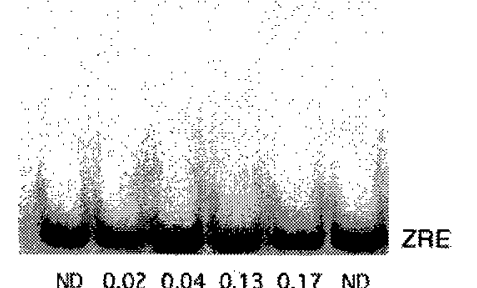

post-transfection, cells were harvested and lysed in Reporter Lysis buffer (Promega, Madison, WI). Transfected cell lysates $(20 \mu \mathrm{l})$ were analyzed for luciferase activity using the luciferase assay system (Promega, Madison, WT) and for $\beta$-galactosidase activity using the Galactolight Plus Chemiluminescent Reporter Assay (BD Biosciences). Each transfection was repeated a minimum of 3 times in duplicate.

\section{Yeast Two-hybrid Interaction Assay}

pGADT7-ZBRK1 5ZFC and its truncation (6ZFC, 7ZFC, 8ZFC, 0ZFC 5ZFC $\Delta 1,5 Z F C \triangle 2$, and 5ZFC $\Delta 3$ ) and broken finger (BF5, BF6, BF7, and $\mathrm{BF} 8$ ) derivatives were individually co-transformed along with $\mathrm{pG}$ BKT7-BRCA1 (expressing BRCA1 amino acids 341-748) into the yeast strain Y187 (BD Biosciences/Clontech). After selection, colonies were expanded in liquid culture for assay of $\beta$-galactosidase activity following previously established procedures (39).

\section{Transient Expression Analysis}

Steady-state levels of GAL4-ZBRK1 5ZFC protein and its truncation and substitution derivatives were comparatively analyzed by immunoblot analysis of transfected whole cell extracts in order to verify equivalent levels of ectopic protein expression. Briefly, U2OS cells transfected with GAL4-ZBRK1 5ZFC, truncation mutants GAL4-ZBRK1 $6 Z$, 7ZFC, 8ZFC, 0ZFC, 5ZFC $\Delta 1,5 Z F C ~ \Delta 2$, and 5ZFC $\Delta 3$, and broken finger mutants GAL4-ZBRK1 5ZFC BF5, BF6, BF7, and BF8 were lysed in Laemmli sample buffer, resolved by SDS-10\% PAGE, and subjected to immunoblot analysis using an antibody directed against the GAL4 DNA-binding domain (sc-510, Santa Cruz Biotechnology, Santa Cruz, CA) and, as an internal control protein, the $\mathrm{p} 89$ subunit of TFIIH (sc-293, Santa Cruz Biotechnology, Santa Cruz, CA). Immunodetection was performed using ECL Western blotting detection reagents (Amersham Biosciences).

\section{RESULTS}

Identification of DNA-binding Determinants on ZBRK1Previously, we demonstrated a strict requirement for BRCA1 in ZBRK1 repression. Specifically, we showed that ZBRK1 repression function was similarly abrogated by genetic ablation of Brca1 or by deletion of the BRCA1-binding domain on ZBRK1 (39). The BRCA1-binding domain on ZBRK1 includes the last four of eight ZBRK1 zinc fingers (zinc fingers 5-8) along with the ZBRK1 $\mathrm{C}$ terminus (39). Whether and how this zinc finger domain contributes to the sequence-specific DNA binding activity of ZBRK1, however, is presently unknown.
Because the specification of individual zinc fingers required to bind to DNA and/or BRCA1 could illuminate the underlying mechanism(s) by which BRCA1 mediates ZBRK1 repression, we initially sought to establish both the number and identity of the ZBRK1 zinc fingers required to bind DNA and BRCA1.

With respect to DNA binding, we showed previously (39) that the eight central ZBRK1 zinc fingers collectively recognize a 15 -bp consensus sequence, GGG $x x x$ CAG $x x x$ TTT. Based on the observation that one $\mathrm{C}_{2} \mathrm{H}_{2}$ zinc finger can bind to $-3 \mathrm{bp}$ of DNA (52-54), only five of the eight ZBRK1 zinc fingers would be predicted to bind to its 15 -bp consensus sequence. To test this prediction, we analyzed a panel of ZBRK1 zinc finger deletion derivatives for their respective abilities to bind to the consensus ZBRK1 DNA-binding site in an EMSA.

To this end, we expressed ZBRK1 as a maltose-binding protein (MBP) chimera in $E$. coli, which permitted the purification of otherwise insoluble ZBRK1 protein. Full-length MBPZBRK1 is expressed poorly, whereas MBP-ZBRK1 $\triangle \mathrm{K}$ (a deletion derivative lacking the $\mathrm{N}$-terminal 143 amino acids of the 532-amino acid full-length ZBRK1 protein) is abundantly expressed. ZBRK1 $\Delta \mathrm{K}$ lacks the $\mathrm{N}$-terminal $\mathrm{KRAB}$ domain but retains the ZBRK1 zinc fingers and the $\mathrm{C}$ terminus, elements that are required for binding to both DNA and/or BRCA1 (Fig. $1, A$ and $B$ ). We have therefore utilized this recombinant ZBRK1 derivative as the background into which truncation and substitution mutations have been introduced for purposes of DNA binding assays.

In an EMSA, MBP-ZBRK1 $\Delta \mathrm{K}$ produced a discrete nucleoprotein complex on a double-stranded oligonucleotide probe corresponding to the consensus ZBRK1-response element (ZRE); a molar excess of unlabeled WT ZRE probe (WT probe), but not a mutant probe (Mut probe), efficiently competed for the formation of this complex, thus establishing sequence-specific DNA binding by MBP-ZBRK1 $\Delta \mathrm{K}$ in this assay (Fig. 1C). To determine the number and identity of the ZBRK1 zinc fingers required to bind to its consensus sequence, we analyzed a series of $\mathrm{C}$-terminal truncation derivatives bearing stepwise deletions of individual ZBRK1 zinc fingers (Fig. 1, $A$ and $B$ ). In 
pGADT7-ZBRK1 derivatives:

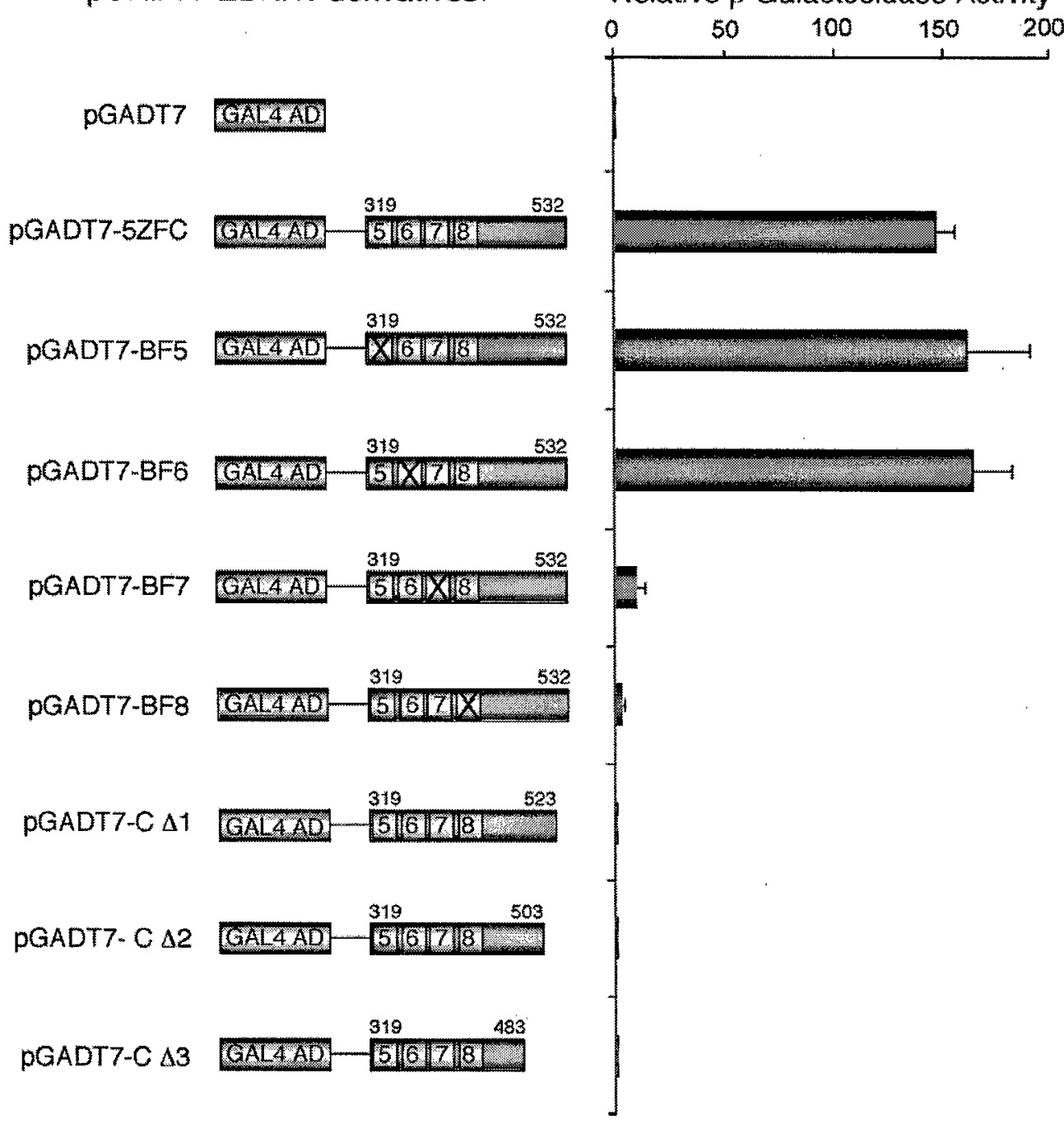

FIG. 3. Identification of BRCA1-binding determinants on ZBRK1. The ZBRK1-binding region on BRCA1 (amino acids 341-748) (39), expressed as a GAL4 DNA-binding domain fusion protein (in the plasmid pGBKT7), was tested for interaction with the indicated fragments of ZBRK1 fused to the GAL4 transactivation domain (plasmid pGADT7) in yeast two-hybrid assays. $\beta$-Galactosidase activities were quantified as described previously (39). Corresponding $\beta$-galactosidase activities obtained with each pGADT7-ZBRK1 5ZFC derivative are expressed relative to that observed with the backbone pGADT7 expression vector alone, which was arbitrarily assigned a value of 1 . Values represents the average of three independent assays, each performed in triplicate, and error bars represent the mean \pm S.D. As a comparative measure of interaction strength, the average $\beta$-galactosidase activity obtained with pGBKT7-p53 and pGADT7-Large T antigen was 512 \pm 13.5 . The validity of two-hybrid interactions observed in these experiments was further substantiated by the following controls. First, we confirmed that the observed $\beta$-galactosidase activities were dependent upon BRCA1 sequences expressed from the plasmid pGBKT7-BRCA1 by performing a parallel series of interaction assays with individual pGADT7-ZBRK1 5ZFC derivatives and the backbone vector pGBKT7 as a negative control. Second, immunoblot analyses of yeast whole cell extracts confirmed that each of the pGADT7-ZBRK1 5ZFC fusion proteins was expressed at roughly equivalent levels, thus excluding the possibility that differences in $\beta$-galactosidase activities derive from difference in fusion protein expression.

$100 \mathrm{~mm} \mathrm{NaCl}$, truncation of the ZBRK1 C terminus (MBPZBRK1 $\triangle \mathrm{K} 8 \mathrm{ZF}$ ) did not appreciably affect DNA binding relative to intact MBP-ZBRK1 $\Delta \mathrm{K}$ (Fig. $1 D$ ). Interestingly, deletion of the eighth and last ZBRK1 zinc finger along with the $\mathrm{C}$ terminus (MBP-ZBRK1 $\triangle \mathrm{K}$ 7ZF) led to an increase in DNA binding activity, suggesting that ZBRK1 ZF8, and possibly the $\mathrm{C}$ terminus, constrains sequence-specific DNA binding mediated by the first seven zinc fingers (Fig. $1 D$ ). This effect was exacerbated under more stringent DNA binding conditions (200 mM NaCl) (Fig. $1 E$ ). At $100 \mathrm{~mm} \mathrm{NaCl}$, stepwise truncation of zinc fingers 7 to 5 led to a slight incremental reduction in sequence-specific DNA binding activity (Fig. $1 D$ ). ZBRK1 derivatives bearing less than four zinc fingers failed to bind to DNA, thereby establishing zinc fingers $1-4$ as the minimal ZBRK1 DNA-binding domain under these conditions (Fig. $1 D$ ). Identical results were observed at $50 \mathrm{~mm} \mathrm{NaCl}$ (data not shown). At $200 \mathrm{~mm} \mathrm{NaCl}$, zinc fingers 1-5 were required for stable DNA binding, and the inclusion of zinc fingers 6 and 7 incrementally stabilized binding (Fig. $1 E$ ).

To examine more rigorously the role of zinc fingers 5-8 in sequence-specific DNA binding by ZBRK1, we examined a set of "broken finger" mutants bearing substitution mutations within each of these zinc fingers. This approach permitted us to assess the individual contribution of each finger within the BRCA1-binding domain to overall DNA binding activity in the context of the eight-fingered ZBRK1 $\Delta \mathrm{K}$ protein and thereby circumvent potential artifacts arising from analyses of truncation mutants. Each broken finger mutant bears a His-to-Asn substitution mutation at the first of the two conserved His residues within the targeted $\mathrm{C}_{2} \mathrm{H}_{2}$ zinc finger (Fig. $2, A$ and $B$ ). The relative conservative nature of this substitution eliminates zinc coordination within the targeted finger, thereby disrupting 
A

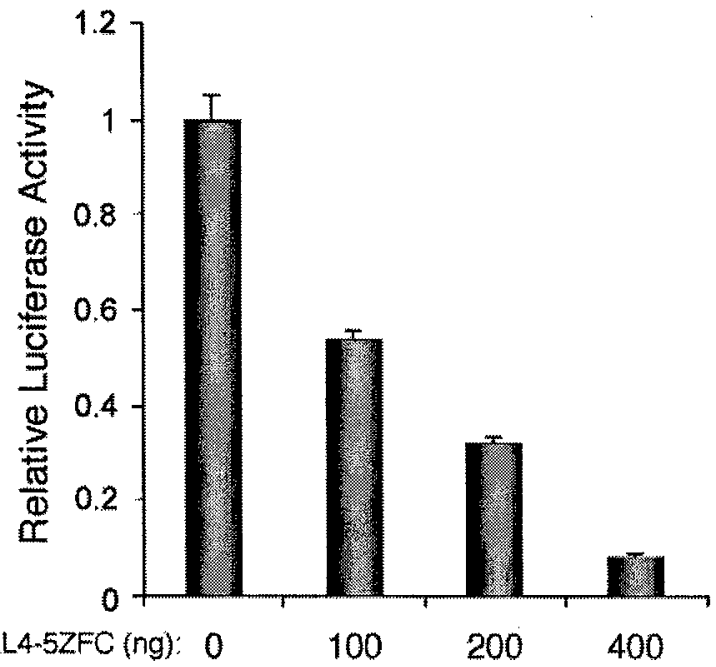

C

Repressor:

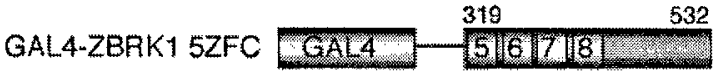

B

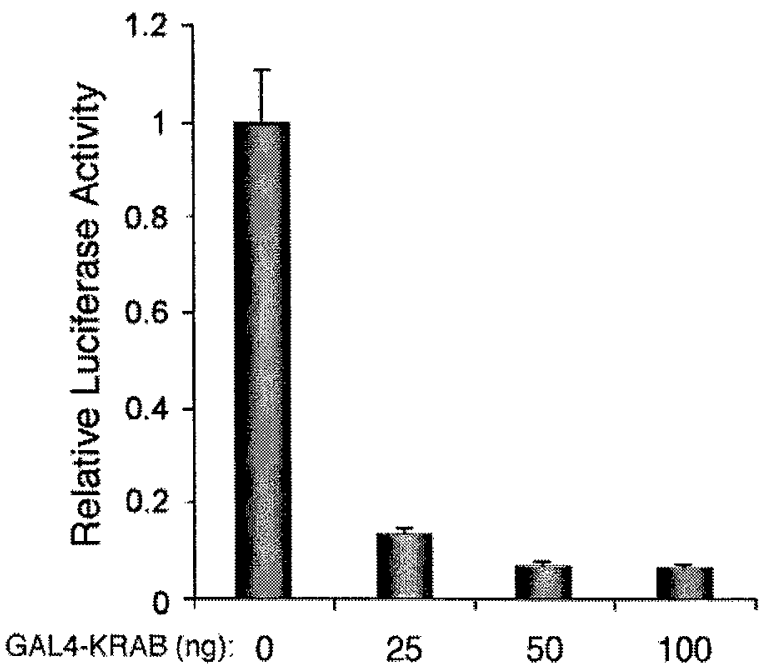

Repressor:

GAL4-ZBRKI KAAB CAL4 KABB

Reporter:

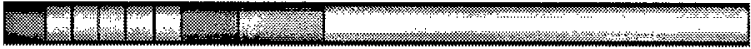

$5 \times G A L 4$

TK

Luciferase

FIG. 4. ZBRK1 harbors two independent transcriptional repression domains. $A$ and $B$, human U2OS cells were transfected with $30 \mathrm{ng}$ of $\mathrm{pG}_{5}$ TK-Luc bearing five copies of the GAL4 DNA-binding site sequence upstream of the TK promoter without or with the indicated nanogram amounts of GAL4-ZBRK1 5ZFC $(A)$ or GAL4-KRAB $(B)$. In this and all subsequent transfection experiments involving effector plasmid titrations, the total amount of DNA in each transfection was fixed by reciprocal titration of the corresponding backbone expression plasmid. Also, in this and all subsequent transfection experiments, the relative luciferase activity represents the ratio of the luciferase activity obtained in a particular transfection to that obtained in cells transfected with only the reporter and pM (GAL4 DNA-binding domain) expression vectors alone. Luciferase activities were first normalized to $\beta$-galactosidase activity obtained by co-transfection of the SV40- $\beta$-gal vector (15 ng) as described previously (39). Error bars represent the S.D. from the average of at least three independent transfections performed in duplicate. $C$, schematic representation of GAL4-ZBRK1 5ZFC and GAL4-ZBRK1 KRAB chimeras (amino acid sequences fused to the GAL4 DNA-binding domain are indicated numerically above each chimera) and the $\mathrm{pG}_{5}$ TK-Luc reporter template used in transfection assays.

its local structure with little effect on the integrity of the remainder of the protein $(55,56)$. Consistent with the results obtained using C-terminal truncation mutants, analysis of individual $B F$ mutants 5-8 revealed zinc finger 5 to be an important ZBRK1 determinant for stable DNA binding, whereas zinc fingers 6 and 7 promote but are not essential for binding (Fig. 2C). Disruption of zinc finger 8 did not appreciably affect the DNA binding activity of MBP-ZBRK1 $\Delta \mathrm{K}$, suggesting that zinc finger 8 is largely dispensable for stable association with DNA (Fig. 2C). Furthermore, local disruption of zinc finger 8 did not relieve constraints on the DNA binding activity of MBP-ZBRK1 $\triangle \mathrm{K}$, suggesting that the $\mathrm{C}$ terminus of ZBRK1 can also mask the inherent DNA binding activity of ZBRK1 zinc fingers 1-7 (Fig. 2C). In summary, the results of DNA-binding analyses delimit the core ZBRK1 DNA-binding domain to zinc fingers 1-4; these zinc fingers are minimally required for stable DNA binding under relatively non-stringent conditions of ionic strength. Zinc finger 5 is a critical and context-dependent determinant of stable binding and represents the extent of the minimal DNA-binding domain under more stringent binding conditions. Zinc fingers 6 and 7, although nonessential, nonetheless further stabilize DNA binding mediated by zinc fingers 1-5. Finally, zinc finger 8 and the $C$ terminus apparently destabilize the maximum potential DNA binding activity inherent in zinc fingers 1-7.

Identification of BRCA1-binding Determinants on ZBRK1Next, we sought to establish more precisely the molecular determinants on ZBRK1 required for BRCA1 binding. Previously, we mapped the BRCA1-binding domain on ZBRK1 to encompass a broad region extending from zinc finger 5 through the $\mathrm{C}$ terminus (5ZFC) (39). To more narrowly define the BRCA1-binding determinants on ZBRK1, we examined the contribution of individual zinc fingers 5-8 as well as sequences within the ZBRK1 $\mathrm{C}$ terminus to BRCA1 binding using a yeast two-hybrid interaction assay. To this end, individual substitution and truncation mutations within ZBRK1 5ZFC were translationally fused to the GAL4 transactivation domain and tested for their respective abilities to bind to the ZBRK1-interaction domain on BRCA1 (amino acids 341-748), translationally fused to the GAL4 DNAbinding domain in yeast (Fig. 3). Corresponding $\beta$-galactosidase activities identified critical determinants of BRCA1 interaction on ZBRK1 to include the $\mathrm{C}$ terminus as well as zinc fingers 7 and 8 (Fig. 3). Deletion of only 9 amino acids from the $\mathrm{C}$ terminus significantly compromised BRCA1 binding, indicating that the unique $\mathrm{C}$ terminus on ZBRK1 is required in its entirety for efficient interaction with BRCA1 (Fig. 3). This observation sug- 
A

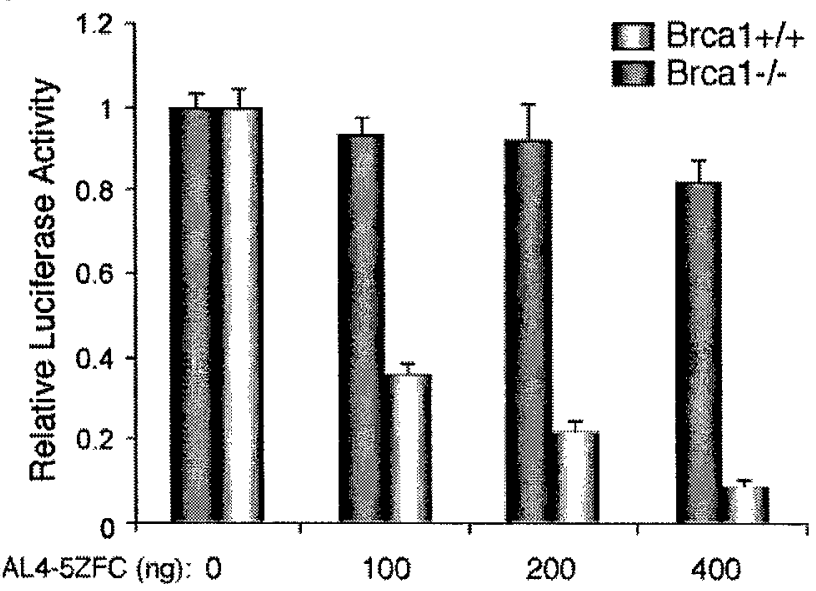

C

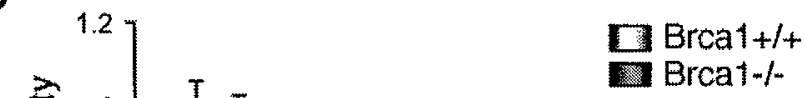

B
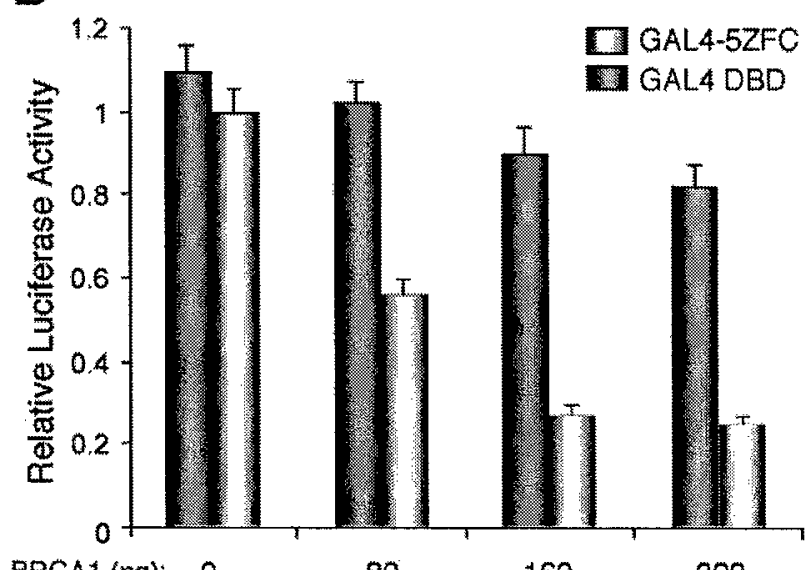

BRCA1 (ng): $0 \quad 80 \quad 160 \quad 320$

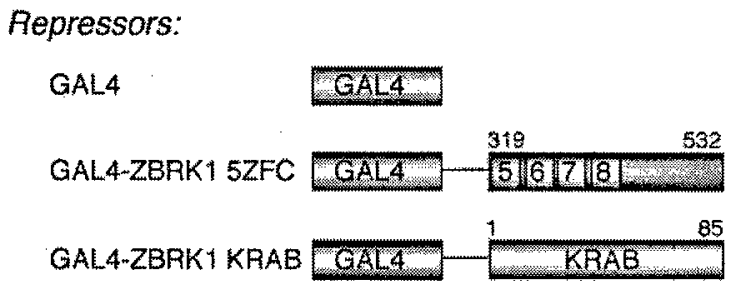

Reporter:

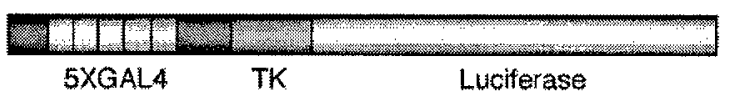

GAL4-KRAB (ng): 0

25

50

100

FIG. 5. The ZBRK1 5ZFC and KRAB repression domains function in a BRCA1-dependent and BRCA1-independent manner, respectively. A, Brca1+I+ and Brca1-1- MEF cells (39) as indicated were transfected with $100 \mathrm{ng}$ of $\mathrm{pG}_{5}$ TK-LUC without or with the indicated nanogram amounts of GAL4-ZBRK1 5ZFC. $B, B r c a 1-/-$ MEF cells were transfected with $100 \mathrm{ng}$ of $\mathrm{pG}_{5}$ TK-Luc and $20 \mathrm{ng}$ of either pM (expressing the GAL4 DNA-binding domain ( $D B D$ ) alone) or GAL4-ZBRK1 5ZFC, respectively, without or with the indicated nanogram amounts of pCS2+BRCA1 expressing wild-type human BRCA1. C, Brca1+1+ and Brca1-1- MEF cells as indicated were transfected with $100 \mathrm{ng}$ of pG ${ }_{5}$ TK-Luc without or with the indicated nanogram amounts of GAL4-ZBRK1 KRAB. A-C, relative luciferase activities were calculated as described in the legend to Fig. 4. $D$, schematic representation of the GAL4 DNA-binding domain, the GAL4-ZBRK1 5ZFC and GAL4-ZBRK1 KRAB chimeras, and the $\mathrm{pG}_{5}$ TK-Luc reporter template used in transfection assays.

gests that the overall conformation of the $\mathrm{C}$ terminus is likely to be important for BRCA1 interaction. Whereas ZBRK1 zinc fingers 7 and 8 are critical for BRCA1 interaction, zinc fingers 5 and 6 do not appear to contribute to BRCA1 binding (Fig. 3). Taken together, these results indicate that important BRCA1-binding determinants on ZBRK1 include those that also modulate its sequence-specific DNA binding activity in both a positive (zinc finger 7) and negative (zinc finger 8 and the $C$ terminus) manner.

The BRCA1-binding Domain on ZBRK1 Functions as an Autonomous BRCA1-dependent Transcriptional Repression Domain-The fact that BRCA1 contacts ZBRK1 through surfaces that are not essential but nonetheless modulatory with respect to DNA binding suggests several potential mechanisms by which BRCA1 might mediate transcriptional repression by ZBRK1. First, BRCA1 could mediate ZBRK1 repression, at least in part, by modulating its sequence-specific association with DNA. This possibility is currently under investigation. Alternatively, or additionally, BRCA1 could mediate repression by DNA-bound ZBRK1. This possibility is supported by our previous observation that clinically validated missense mutations within the BRCA1 $\mathrm{C}$ terminus that do not disrupt its interaction with ZBRK1 nonetheless abrogate its ZBRK1 co- repressor activity (39). To test this possibility directly, we examined whether the BRCA1-binding domain on ZBRK1 could function as a BRCA1-dependent transcriptional repression domain when tethered to a heterologous DNA-binding domain. This approach permitted us to assess the influence of BRCA1 on the repression function of ZBRK1 independently of any effects that it might have on the DNA binding activity of ZBRK1. Accordingly, we initially tested the ability of ZBRK1 5ZFC (extending from zinc finger 5 to the $\mathrm{C}$ terminus) to function as an independent repression domain when linked to the GAL4 DNA-binding domain. GAL4-ZBRK1 5ZFC was transiently expressed in U2OS human osteosarcoma cells, and its influence on transcription from a $\mathrm{pG}_{5} \mathrm{TK}$-Luc reporter template bearing five copies of the consensus GAL4 DNA-binding site upstream of the herpes simplex virus (HSV) TK gene promoter was examined. GAL4-ZBRK1 5ZFC conferred greater than 10fold repression upon reporter gene expression in a dose-dependent manner (Fig. 4A). We also confirmed the presence of a potent KRAB repression domain within the ZBRK1 $\mathrm{N}$ terminus by examining its ability repress $\mathrm{pG}_{5}$ TK-Luc reporter gene expression when tethered to the GAL4 DNA-binding domain (Fig. $4 B$ ). Based on quantitative immunoblot analysis of transfected 
A
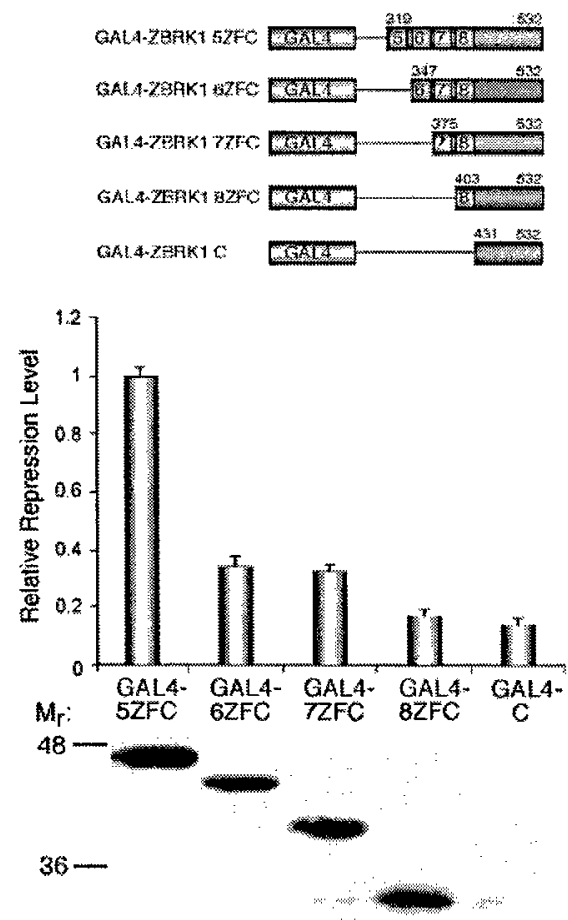

B
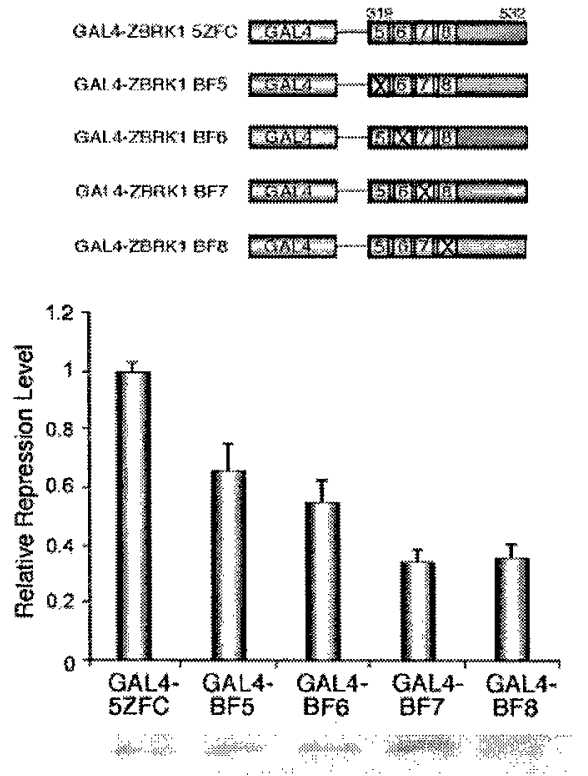

6
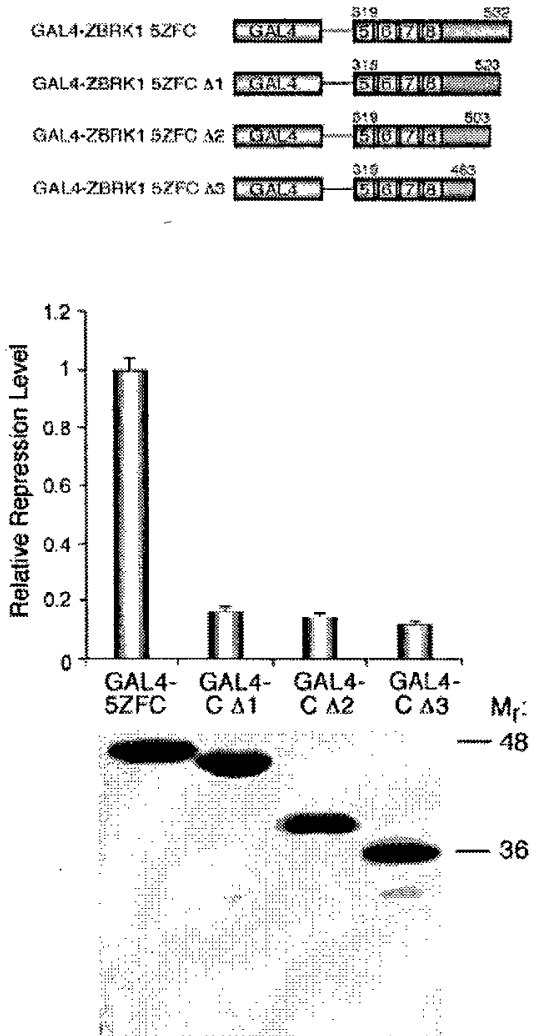

FIG. 6. Functional analysis of ZBRK1 5ZFC truncation and substitution derivatives. $A-C$, U2OS cells were transfected with 30 ng of $\mathrm{pG}_{5}$ TK-Luc and the following amounts of GAL4-ZBRK1 5ZFC or its indicated derivatives: $A, 5 \mathrm{ZFC}, 400 \mathrm{ng}$; $6 \mathrm{ZFC}, 7 \mathrm{ZFC}, 8 \mathrm{ZFC}$, and C, 200 ng; $B$, $5 Z F C$ or its indicated broken finger derivatives, $200 \mathrm{ng} ; C, 5 Z F C, 400 \mathrm{ng} ; \Delta 1,200 \mathrm{ng} ; \Delta 2,400 \mathrm{ng} ; \Delta 3,200 \mathrm{ng}$. In each panel, the relative repression level represents the relative luciferase activity obtained with a particular GAL4-ZBRK1 5ZFC derivative divided by that obtained with GAL4ZBRK1 5ZFC. Relative luciferase activities were calculated as described in the legend to Fig. 4 . In these experiments, the relative luciferase activity observed with GAL4-ZBRK1 5ZFC (three independent transfections performed in duplicate) was as follows: $A$, 0.10 corresponding to 10-fold repression of reporter template activity; $B, 0.21$ corresponding to an approximate 5-fold repression of reporter template activity; $C, 0.08$ corresponding to 12.5-fold repression of reporter template activity. To confirm that GAL4-ZBRK1 5ZFC and its truncation and substitution derivatives are expressed at roughly equivalent levels, lysates from U2OS cells transfected with GAL4-ZBRK1 5ZFC or its derivatives (the same amounts used in functional analysis and indicated above) were resolved by SDS-10\% PAGE and subjected to immunoblot analysis using antibodies specific for the GAL4 DNA-binding domain. Molecular weight marker positions $\left(M_{\mathrm{r}}\right)$ are indicated. GAL4-ZBRK1 5ZFC and its derivatives are represented schematically at the top of each panel.

cell extracts, the ZBRK1 KRAB domain, on a molar basis, appears to be a stronger transcriptional repression domain than the ZBRK1 BRCA1-binding domain (data not shown). Nonetheless, this result indicates the presence within ZBRK1 of two portable transcriptional repression domains, an N-terminal KRAB domain and a novel C-terminal transcriptional repression domain (5ZFC) that encompasses the BRCA1-binding domain.

To confirm the requirement for BRCA1 in transcriptional repression by ZBRK1 $5 \mathrm{ZFC}$, we tested the repression function of GAL4-ZBRK1 5ZFC in Brca1+/ + and Brca 1-/- MEF cells. GAL4-ZBRK1 5ZFC conferred up to 10-fold repression in Brca1+/+ MEFs, whereas little or no repression activity was observed in Brca1-/- MEFs (Fig. 5A). Ectopic expression of BRCA1 in Brca1-1- MEFs restored ZBRK1 5ZFC-directed transcriptional repression (Fig. $5 B$ ), establishing conclusively that BRCA1 mediates repression by DNA-bound ZBRK1 5ZFC. In contrast to ZBRK1 $5 \mathrm{ZFC}$, the ZBRK1 KRAB domain repressed transcription equivalently in both $\mathrm{Brca} 1+/+$ and Brca1-/- MEFs (Fig. 5C). Thus, the N-terminal KRAB and C-terminal 5ZFC repression domains within ZBRK1 can be distinguished functionally on the basis of their respective requirements for BRCA1.

BRCA1-binding Is Necessary but Not Sufficient for ZBRK1
5ZFC Repression Function-To more narrowly define the boundaries of the BRCA1-dependent $5 \mathrm{ZFC}$ repression domain within ZBRK1, we examined a panel of ZBRK1 5ZFC truncation and substitution mutants for their respective repression activities in vivo. Relative to the intact 5ZFC domain, deletion or disruption of zinc fingers 5 or 6 individually reduced repression activity by $2-3$-fold (Fig. $6, A$ and $B$ ), whereas individual deletion or disruption of zinc fingers 7 or 8 reduced repression activity by $3-6$-fold (Fig. $6, A$ and $B$ ). These results indicate that zinc fingers 5-8 are all required for the integrity of the 5ZFC repression domain, although zinc fingers 7 and 8 appear to be quantitatively more important. Deletion of only 9 amino acids from the $\mathrm{C}$ terminus of ZBRK1 severely compromised the repression function of the $5 \mathrm{ZFC}$ domain, indicating that the entire $\mathrm{C}$ terminus is likely to be important for $5 \mathrm{ZFC}$ repression activity (Fig. $6 \mathrm{C}$ ). Thus, the $5 \mathrm{ZFC}$ repression domain extending from ZBRK1 zinc finger 5 through the $\mathrm{C}$ terminus appears to constitute an intact repression domain that cannot be further delimited. This analysis also reveals an imperfect correlation between BRCA1 binding and transcriptional repression by the 5ZFC repression domain. Thus, disruption of ZBRK1 zinc fingers 7 or 8 or truncation of the $C$ terminus severely compromised BRCA1 binding (Fig. 3) and transcriptional repression (Fig. 6). By contrast, disruption of zinc fingers 5 or 6 , which are 
A \begin{tabular}{|}
\hline D $0 \mathrm{mM}$ TSA \\
B330 mM TSA \\
\hline
\end{tabular}

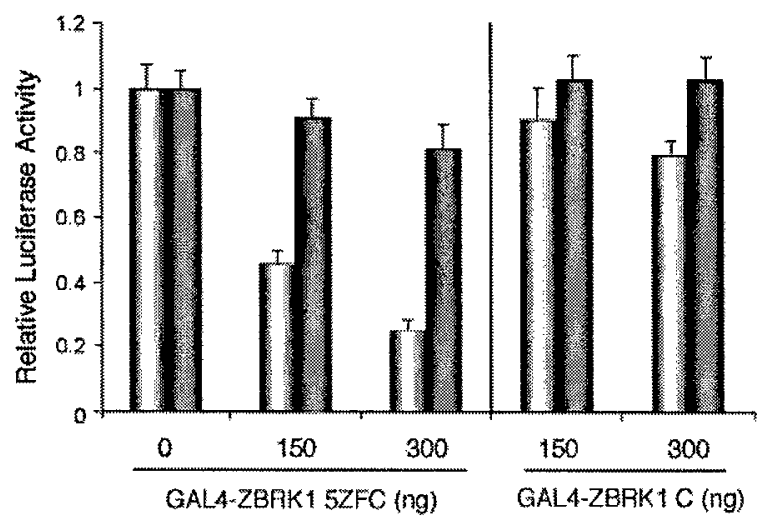

B

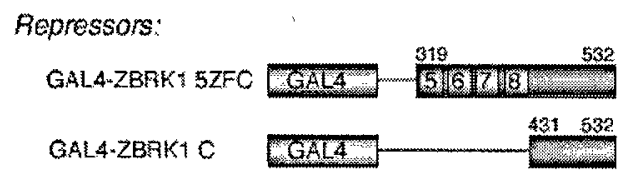

Reporter:

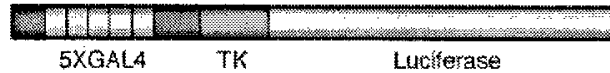

FIG. 7. ZBRK1 5ZFC-directed repression is reversed by trichostatin A. A, U2OS cells were transfected with $30 \mathrm{ng}$ of $\mathrm{pG}_{5}$ TK-Luc without or with the indicated nanogram amounts of GAL4-ZBRK1 5ZFC or GAL4-ZBRK1 C. Where indicated, TSA (330 nM) was also included. Relative luciferase activities were calculated as described in the legend to Fig. 4. $B$, schematic representation of the GAL4-ZBRK1 5ZFC and GAL4-ZBRK1 $\mathrm{C}$ chimeras and the $\mathrm{pG}_{5} \mathrm{TK}$-Luc reporter template used in transfection assays.

not required for BRCA1 binding (Fig. 3), nonetheless significantly compromised transcriptional repression (Fig. 6, $A$ and $B$ ). On this basis we conclude that BRCA1 binding is necessary but not sufficient for $5 \mathrm{ZFC}$ repression function. This suggests that ZBRK1 zinc fingers 5 and 6 may possibly contact an additional co-repressor(s).

The BRCA1-dependent 5ZFC Repression Domain Is Histone Deacetylase-dependent and Promoter-specific-Previously, BRCA1 has been shown to interact through its C-terminal BRCT repeats with histone deacetylases (HDACs) 1 and 2 (57). HDACs remove acetyl groups from lysine residues on histone tails and thus promote the formation of transcriptionally repressive chromatin. To determine the contribution of HDAC activity to repression mediated by ZBRK1 5ZFC, we tested the effect of the selective HDAC inhibitor trichostatin A (TSA) on repression mediated by GAL4-ZBRK1 5ZFC. TSA largely reversed GAL4-ZBRK1 5ZFC repression in U2OS cells, implicating HDAC activity in this process (Fig. 7).

Like the ZBRK1 5ZFC repression domain, KRAB repression domains function through HDACs as well as through histone methyltransferases and heterochromatin proteins (41-47). This prompted us to comparatively examine the promoter specificities of the ZBRK1 $5 \mathrm{ZFC}$ and $\mathrm{KRAB}$ repression domains. The ZBRK1 N-terminal KRAB domain repressed transcription potently from each of three different RNA polymerase II promoters tested: the SV40 major late, the HSV TK, and the human small nuclear ribonucleoprotein $\mathrm{N}$ (SNRPN) promoters (Fig. 8B). By contrast, ZBRK1 5ZF5C repressed transcription potently from the HSV TK promoter, moderately from the SV40 promoter, and not at all from the SNRPN promoter (Fig. 8A). These results indicate that the ZBRK1 KRAB and 5ZFC repression domains can be distinguished functionally not only by their requirement for BRCA1 but also on the basis of their promoter specificities; whereas the ZBRK1 KRAB repression domain exhibits broad promoter selectivity, the BRCA1-dependent $5 Z F C$ repression domain exhibits a more restricted promoter bias.

\section{DISCUSSION}

A central question regarding the role of BRCA1 in transcription control concerns the means by which it mediates genespecific regulation in the absence of sequence-specific DNA binding activity. In part, this question has been answered by the identification of a growing number of sequence-specific DNA-binding transcription factors with which BRCA1 physically and functionally interacts. In this regard, our previous identification of ZBRK1 as a BRCA1-dependent transcriptional repressor provided a molecular basis to link BRCA1 directly to the regulation of $G A D D 45 a$ gene expression (39). Other work has rendered it clear that the BRCA1 regulation of $G A D D 45 a$ gene transcription is likely to be complex and mediated not only through ZBRK1 but other trans-acting factors, including OCT1 and NF-YA $(21,32,39)$. Presently, however, little is known regarding the mechanism(s) by which BRCA1 mediates sequence-specific transcriptional control through the various transcription factors with which it interacts. Here we have investigated the functional interaction between ZBRK1 and BRCA1 in an effort to understand the role of BRCA1 in sequence-specific transcriptional repression.

Our studies suggest that BRCA1 mediates ZBRK1 repression, at least in part, through its targeted recruitment to a novel C-terminal repression domain (5ZFC) within ZBRK1. Structurally, this repression domain comprises the last four zinc fingers and the unique C-terminal extension of ZBRK1. The identification of 5ZFC as a discrete functional domain was revealed by its ability to repress transcription when tethered to a heterologous DNA-binding domain (Fig. 4) and its functional resistance to truncation or substitution mutagenesis (Fig. 6). Importantly, we demonstrated that 5ZFC repression function is dependent upon BRCA1; genetic ablation of Brca 1 or disruption of BRCA1-binding determinants on 5ZFC similarly abrogates the repression function of this domain (Figs. 5 and 6). The functional contribution of this domain to BRCA1-dependent ZBRK1 repression is reflected by our previous observation that deletion of the ZBRK1 $\mathrm{C}$ terminus abrogates ZBRK1 repression through natural ZBRK1-response elements (39). However, whereas BRCA1 binding is necessary, our studies here suggest that it is not sufficient for 5ZFC-directed repression. First, BRCA1 binding and repression determinants within this domain can be separated, suggesting a possible functional requirement for a co-repressor(s) in addition to BRCA1 (Figs. 3 and 6). Second, 5ZFC-directed repression is HDAC-dependent (Fig. 7). Thus, we propose that the ZBRK1 5ZFC repression domain recruits BRCA1 as part of a higher order repression complex that minimally includes an associated HDAC activity. Targeted attempts to identify the functionally relevant BRCA1-associated co-repressor activities are currently underway.

Our work further reveals unique insight into the structural and functional organization of ZBRK1, a member of the KRABZFP family. The $\sim 220$ members of this family make up a significant proportion of the transcription factor complement of the human proteome and are believed to occupy important regulatory roles in development, differentiation, and transformation (41, 58-63). Despite their potential biological signifirance, our current understanding of the mechanisms through 


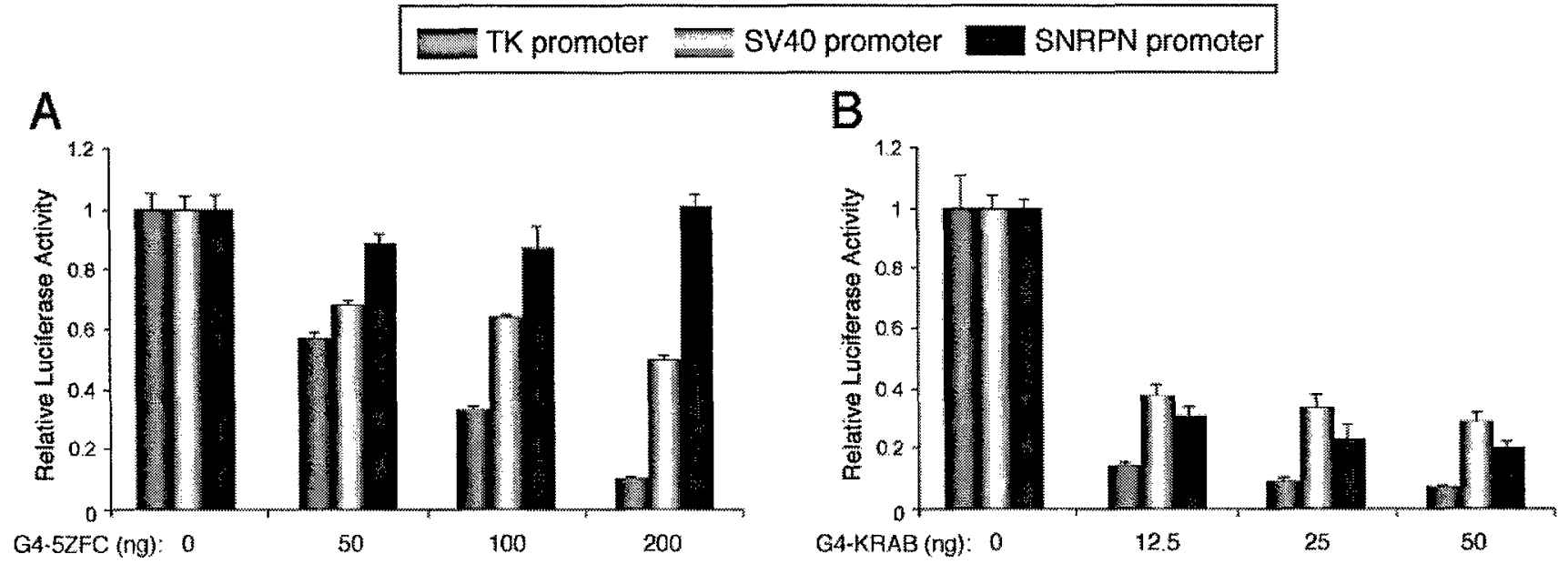

C

Repressor:

GAL4-ZBRK1 5ZFC

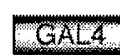

319

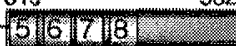

Repressor:

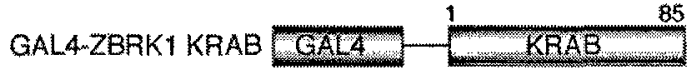

Reporters:

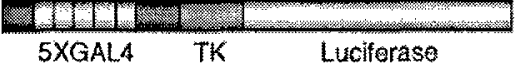

$5 \times$ GAL4

TK

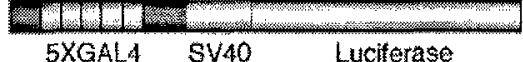

5XGALA SV40 Luciferase

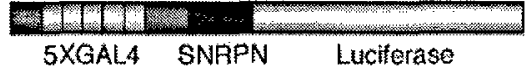

FIG. 8. The ZBRK1 KRAB and 5ZFC repression domains exhibit unique promoter specificities. $A$ and $B$, U2OS cells were transfected

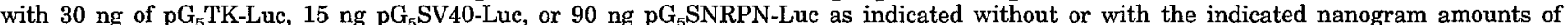
GAL4-ZBRK1 5ZFC $(A)$ or GAL4-ZBRK1 KRAB $(B)$. Relative luciferase activities were calculated as described in the legend to Fig. 4. $C$, schematic representation of the GAL4-ZBRK1 KRAB and GAL4-ZBRK1 5ZFC chimeras, and the pG $_{5}$ TK-Luc, pG $_{5}$ SV40-Luc, and pG ${ }_{5}$ SNRPN-Luc reporter templates used in transfections.

which individual members of this protein family function is still rather limited. Thus, although considerable mechanistic insight into the repression function of the KRAB domain has been revealed in recent years (41-47), comparatively little is known regarding the role of KRAB domain-associated zinc fingers in transcriptional repression apart from their presumed role in sequence-specific DNA binding. In part, this gap in knowledge derives from the limited availability of KRAB-ZFP target sequences with which structure-function analyses may be carried out. In the case of several KRAB-ZFPs whose corresponding binding site sequences have been identified, an additional function(s) for individual zinc fingers beyond DNA binding seems implicit. For example, based on the observation that one $\mathrm{C}_{2} \mathrm{H}_{2}$ zinc finger can bind to $-3 \mathrm{bp}$ of DNA (52-54), the established target sequence lengths of 5 and $27 \mathrm{bp}$, respectively, for the 8- and 10-fingered ZNF202 and KS1 proteins are incompatible with DNA contact mediated by every zinc finger $(61,63)$. Our previous derivation of a consensus binding sequence for ZBRK1 has permitted us here to dissect a long array KRAB-ZFP and examine the contribution of individual zinc fingers to both sequence-specific DNA-binding and transcriptional repression. Our studies reveal the ZBRK1 zinc fingers to be multifunctional in nature, with dedicated roles in binding DNA, BRCA1, or both.

First, zinc fingers 1-4 are essential for DNA binding activity and compose the minimal DNA-binding domain under moderate conditions of ionic strength (Fig. 1). Zinc finger 5 appears to be a critical and context-dependent DNA-binding zinc finger; this finger represents the extent of the minimal DNA-binding domain under more stringent conditions (Fig. 1). Zinc fingers 6 and 7 are not essential for DNA binding but nonetheless enhance the stability of DNA binding (Figs. 1 and 2). Finally, zinc finger 8 (along with the $\mathrm{C}$ terminus) is dispensable for, and may possibly destabilize, DNA binding mediated by zinc fingers 1-7 (Fig. 1). Taken together, these findings reveal the ZBRK1 zinc fingers to compose at least two functional classes: those that make minimal essential contacts with DNA (fingers 1-4) and those that modulate the stability of these contacts (fingers 5-8). Importantly, zinc fingers 5-8 that modulate ZBRK1 DNA binding activity also represent critical determinants of repression by DNA-bound ZBRK1 through association with co-repressors, including BRCA1. These findings thus extend the established role of KRAB-zinc fingers to include protein-protein interactions critical for transcriptional repression, and also identify within ZBRK1 dual specificity zinc fingers with twin roles in DNA-binding and transcriptional repression.

Our work advances the understanding of DNA recognition by KRAB-ZFPs in several respects. First, we provide further empirical evidence to support predictive models for $\mathrm{C}_{2} \mathrm{H}_{2}$ zine finger-DNA recognition. Structural studies of 3- and 5-fingered proteins in complex with DNA have indicated that individual zinc fingers bind to $\sim 3$ bp of DNA (52-54). Based on this model, five of the eight ZBRK1 zinc fingers would be predicted to bind to its 15 -bp recognition sequence. In fact, DNA-binding analyses revealed that under mild conditions of ionic strength, the first four ZBRK1 zinc fingers are sufficient to confer stable binding to its consensus sequence. However, more stringent conditions unmasked a requirement for the fifth finger, consistent with the aforementioned structural models. Second, our identification of ZBRK1 zinc finger 5 as a critical and context- 
dependent DNA-binding determinant could clarify recent issues concerning selectivity among KRAB-ZFPs that recognize overlapping DNA-binding site sequences. In this regard, a fourfingered KRAB-ZFP called SZF1 was recently shown to recognize a DNA-binding site in common with ZBRK1 (64). The observation that SZF1 and ZBRK1 exhibit overlapping DNAbinding specificities in vitro raises the possibility that these proteins might compete for a common binding site(s) in vivo (64). This, in turn, could have significant implications for the biological regulation of target gene transcription by each of these proteins. However, as we show here, ZBRK1 zinc finger 5 is a critical DNA-binding determinant under more stringent conditions of increased ionic strength and also increased nonspecific competitor concentrations in vitro (Fig. 1 and data not shown). Because these conditions are more likely to approximate those of the cellular milieu, in which target site location must be achieved in the presence of a vast excess of like and unlike DNA sequences, ZBRK1 zinc finger 5 could represent a critical determinant of target site selection in vivo. Beyond zinc finger 5, zinc fingers 6 and 7 through enhanced affinity and/or protein-protein interactions could further influence ZBRK1 target site selectivity.

Our identification within ZBRK1 of a C-terminal BRCA1-dependent repression domain in addition to the $\mathrm{N}$-terminal $\mathrm{KRAB}$ domain represents the first demonstration of a KRABZFP harboring two independent repression domains. More importantly, the presence of two inherent repression domains could have important implications for gene-specific transcription control by ZBRK1. As we show here, the KRAB and Cterminal repression domains within ZBRK1 can be distinguished functionally on the basis of their respective requirements for $\mathrm{BRCA1}$; the $\mathrm{C}$-terminal repression domain is BRCA1-dependent, whereas the KRAB domain is not. This functional distinction may in part underlie the unique promoter specificities of the two repression domains. Whereas the KRAB repression domain exhibits broad promoter specificity, the BRCA1-dependent repression domain exhibits a more restricted promoter bias. Thus, the relative contribution of the BRCA1-dependent repression domain to overall ZBRK1 repression may vary among different ZBRK1 target promoters, effectively expanding the regulatory potential available at ZBRK1 target genes. It will be of interest in future studies to determine whether and how these discrete repression domains function synergistically to confer ZBRK1 repression.

Finally, although our work suggests that BRCA1 mediates repression by DNA-bound ZBRK1, we cannot exclude the additional possibility that BRCA1 also mediates ZBRK1 repression, at least in part, by modulating it sequence-specific DNA binding activity. Our observation that the BRCA1-binding surface on ZBRK1 includes zinc fingers that modulate its DNA binding activity in both a positive (zinc finger 7 ) and negative (zinc finger 8) manner is consistent with this possibility, and studies are currently underway to address this important issue. Nonetheless the studies presented here shed new light on the functional organization of ZBRK1 as a model KRAB-ZFP and further define the role of BRCA1 in sequence-specific transcription control.

Acknowledgments-We thank our laboratory colleagues for advice and discussion and P. Renee Yew for insight and comments. We thank Drs. Y. Tony Ip (University of Massachusetts Medical School, Worcester, MA) and Paul A. Wade (Emory University School of Medicine, Atlanta, GA) for reporter plasmids.

\section{REFERENCES}

1. Miki, Y., Swensen, J., Shattuck-Eidens, D., Futreal, P. A., Harshman, K., Tavtigian, S., Liu, Q., Cochran, C., Bennett, L. M., Ding, W., Bell, R., Rosenthal, J., Hussey, C., Tran, T., McClure, M., Frye, C., Hattier, T. Phelps, R., Haugen-Strano, A., Katcher, H., Yakumo, K., Gholami, Z.,
Shaffer, D., Stone, S., Bayer, S., Wray, C., Bogden, R., Dayananth, P., Ward, J., Tonin, P., Narod, S., Bristow, P. K., Norris, F. H. Helvering, L, Morrison, P., Rosteck, P., Lai, M., Barrett, J. C., Lewis, C., Neuhausen, S., Cannon-Albright, L., Goldgar, D., Wiseman, R., Kamb, A., and Skolnick, M. H. (1994) Science 266, 66-71

2. Hall, J. M., Lee, M. K., Newman, B., Morrow, J. E., Anderson, L. A., Huey, B., and King, M. C. (1990) Science 250, 1684-1689

3. Wooster, R., and Weber, B. L. (2003) N. Engl. J. Med. 348, 2339-2347

4. Tirkkonen, M., Johannsson, O., Agnarsson, B. A., Olsson, H., Ingvarsson, S., Karhu, R., Tanner, M., Isola, J., Barkardottir, R. B., Borg, A., and Kallioniemi, O. P. (1997) Cancer Res. 57, 1222-1227

5. Venkitaraman, A. R. (2002) Cell 108, 171-182

6. Zheng, L., Li, S., Boyer, T. G., and Lee, W. H. (2000) Oncogene 19, 6159-6175

7. Xu, X., Weaver, Z., Linke, S. P., Li, C., Gotay, J., Wang, X. W., Harris, C. C., Ried, T., and Deng, C. X. (1999) Mol. Cell 3, 389-395

8. Scully, R., and Livingston, D. M. (2000) Nature 408, 429-432

9. Welcsh, P. L., and King, M. C. (2001) Hum. Mol. Genet. 10, 705-713

10. Deng, C. X. (2001) Mutat. Res. 477, 183-189

11. Rosen, E. M., Fan, S., Pestell, R. G., and Goldberg, I. D. (2003) J. Cell Physiol. 196, 19-41

12. Xu, B., Kim, S., and Kastan, M. B. (2001) Mol. Cell. Biol. 21, 3445-3450

13. Cortez, D., Wang, Y., Qin, J., and Elledge, S. J. (1999) Science 286, 1162-1166

14. Lee, J. S., Collins, K. M., Brown, A. L., Lee, C. H., and Chung, J. H. (2000) Nature 404, 201-204

15. Scully, R., Chen, J., Ochs, R. L., Keegan, K., Hoekstra, M., Feunteun, J., and Livingston, D. M. (1997) Cell 90, 425-435

16. Li, S., Ting, N. S., Zheng, L., Chen, P. L., Ziv, Y., Shiloh, Y., Lee, E. Y., and Lee, W. H. (2000) Nature 406, 210-215

17. Moynahan, M. E., Chiu, J. W., Koller, B. H., and Jasin, M. (1999) Mol. Cell 4, 511-518

18. Zhong, Q., Chen, C. F., Li, S., Chen, Y., Wang, C. C., Xiao, J., Chen, P. L., Sharp, Z. D., and Lee, W. H. (1999) Science 285, 747-750

19. Scully, R., Chen, J., Plug, A., Xiao, Y., Weaver, D., Feunteun, J., Ashley, T., and Livingston, D. M. (1997) Cell 88, 265-275

20. Somasundaram, K., Zhang, H., Zeng, Y. X., Houvras, Y., Peng, Y., Wu, G. S., Licht, J. D., Weber, B. L., and El-Deiry, W. S. (1997) Nature 389, 187-190

21. Harkin, D. P., Bean, J. M., Miklos, D., Song, Y. H., Truong, V. B., Englert, C., Christians, F. C., Ellisen, L. W., Maheswaran, S., Oliner, J. D., and Haber, D. A. (1999) Cell $\mathbf{9 7}, 575-586$

22. Deng, C. X., and Brodie, S. G. (2000) BioEssays 22, 728-737

23. Monteiro, A. N. (2000) Trends Biochem. Sci. 25, 469-474

24. Starita, L. M., and Parvin, J. D. (2003) Curr. Opin. Cell Biol. 15, 345-350

25. Jasin, M. (2002) Oncogene 21, 8981-8993

26. Baer, R., and Ludwig, T. (2002) Curr. Opin. Genet. Dev. 12, 86-91

27. Bochar, D. A., Wang, L., Beniya, H., Kinev, A., Xue, Y., Lane, W. S., Wang, W., Kashanchi, F., and Shiekhattar, R. (2000) Cell 102, 257-265

28. Miyake, T., Hu, Y. F., Yu, D. S., and Li, R. (2000) J. Biol. Chem. 275, 40169-40173

29. MacLachlan, T. K., Somasundaram, K., Sgagias, M. Shifman, Y., Muschel, R. J., Cowan, K. H., and El-Deiry, W. S. (2000) J. Biol. Chem. 275, 2777-2785

30. Aprelikova, O., Pace, A. J., Fang, B., Koller, B. H., and Liu, E. T. (2001) J. Biol. Chem. 276, 25647-25650

31. Welcsh, P. L., Lee, M. K., Gonzalez-Hernandez, R. M., Black, D. J., Mahadevappa, M., Swisher, E. M., Warrington, J. A., and King, M. C. (2002) Proc. Natl. Acad. Sci. U. S. A. 99, 7560-7565

32. Fan, W., Jin, S., Tong, T., Zhao, H., Fan, F., Antinore, M. J., Rajasekaran, B., Wu, M., and Zhan, Q. (2002) J. Biol. Chem. 277, 8061-8067

33. Ouchi, T., Monteiro, A. N., August, A., Aaronson, S. A., and Hanafusa, H. (1998) Proc. Natl. Acad. Sci. U. S. A. 95, 2302-2306

34. Chai, Y. L., Cui, J., Shao, N., Shyam, E,, Reddy, P., and Rao, V. N. (1999) Oncogene 18, 263-268

35. Fan, S., Ma, Y. X., Wang, C., Yuan, R. Q., Meng, Q., Wang, J. A., Erdos, M., Goldberg, I. D., Webb, P., Kushner, P. J., Pestell, R. G., and Rosen, E. M. (2001) Oncogene 20, 77-87

36. Park, J. J., Irvine, R. A., Buchanan, G., Koh, S. S., Park, J. M., Tilley, W. D., Stallcup, M. R., Press, M. F., and Coetzee, G. A. (2000) Cancer Res. 60, $5946-5949$

37. Wang, Q., Zhang, H., Kajino, K., and Greene, M. I. (1998) Oncogene 17, $1939-1948$

38. Zhang, H., Somasundaram, K., Peng, Y., Tian, H., Bi, D., Weber, B. L., and El-Deiry, W. S. (1998) Oncogene 16, 1713-1721

39. Zheng, L., Pan, H., Li, S., Flesken-Nikitin, A., Chen, P. L., Boyer, T. G., and Lee, W. H. (2000) Mol. Cell 6, 757-768

40. Zheng, L., Annab, L. A., Afshari, C. A., Lee, W. H., and Boyer, T. G. (2001) Proc. Natl. Acad. Sci. U. S. A. 98, 9587-9592

41. Collins, T., Stone, J. R., and Williams, A. J. (2001) Mol. Cell. Biol. 21, $3609-3615$

42. Friedman, J. R., Fredericks, W. J., Jensen, D. E., Speicher, D. W., Huang, X. P., Neilson, E. G., and Rauscher, F. J., III (1996) Genes Dev. 10, 2067-2078

43. Kim, S. S., Chen, Y. M., O'Leary, E., Witzgall, R., Vidal, M., and Bonventre, J. V. (1996) Proc. Natl. Acad. Sci. U. S. A. 93, 15299-15304

44. Moosmann, P., Georgiev, O., Le Douarin, B., Bourquin, J. P., and Schaffner, W. (1996) Nucleic Acids Res. 24, 4859-4867

45. Lechner, M. S., Begg, G. E., Speicher, D. W., and Rauscher, F. J., III (2000) Mol. Cell. Biol. 20, 6449-6465

46. Schultz, D. C., Ayyanathan, K., Negorev, D., Maul, G. G., and Rauscher, F. J., III (2002) Genes Dev. 16, 919-932

47. Schultz, D. C., Friedman, J. R., and Rauscher, F. J., III (2001) Genes Dev. 15, $428-443$

48. Kastan, M. B., Zhan, Q., el-Deiry, W. S., Carrier, F., Jacks, T., Walsh, W. V. Plunkett, B. S., Vogelstein, B., and Fornace, A. J., Jr. (1992) Cell 71, 
$587-597$

49. Hollander, M. C., Alamo, I., Jackman, J., Wang, M. G., McBride, O. W., and Fornace, A. J., Jr. (1993) J. Biol. Chem. 268, 24385-24393

50. Sadowski, I., Bell, B., Broad, P., and Hollis, M. (1992) Gene (Amst.) 118, 137-141

51. Fujita, N., Jaye, D. L., Kajita, M., Geigerman, C., Moreno, C. S., and Wade, P. A. (2003) Cell 113, 207-219

52. Pavletich, N. P., and Pabo, C. O. (1991) Science 252, 809-817

53. Pavletich, N. P., and Pabo, C. O. (1993) Science 261, 1701-1707

54. Wolfe, S. A., Nekludova, L., and Pabo, C. O. (2000) Annu. Rev. Biophys. Biomol. Struct. 29, 183-212

55. Del Rio, S., Menezes, S. R., and Setzer, D. R. (1993) J. Mol. Biol. 233, 567-579

56. Del Rio, S., and Setzer, D. R. (1993) Proc. Natl. Acad. Sci. U. S. A. 90, 168-172

57. Yarden, R. I., and Brody, L. C. (1999) Proc. Natl. Acad. Sci. U. S. A. 96, 4983-4988
58. Jheon, A. H., Ganss, B., Cheifetz, S., and Sodek, J. (2001) J. Biol. Chem. 276, 18282-18289

59. Bellefroid, E. J., Poncelet, D. A., Lecocq, P. J., Revelant, O., and Martial, J. A. (1991) Proc. Natl. Acad. Sci. U. S. A. 88, 3608-3612

60. Bellefroid, E. J., Marine, J. C., Ried, T., Lecocq, P. J., Riviere, M., Amemiya, C. Poncelet, D. A., Coulie, P. G., de Jong, P., Szpirer, C., Ward, D. C., and Martial, J. A. (1993) EMBO J. 12, 1363-1374

61. Gebelein, B., and Urrutia, R. (2001) Mol. Cell. Biol. 21, 928-939

62. Gebelein, B., Fernandez-Zapico, M., Imoto, M., and Urrutia, R. (1998) J. Clin. Investig. 102, 1911-1919

63. Wagner, S., Hess, M. A., Ormonde-Hanson, P., Malandro, J., Hu, H., Chen, M., Kehrer, R., Frodsham, M., Schumacher, C., Beluch, M., Honer, C., Skolnick, M., Ballinger, D., and Bowen, B. R. (2000) J. Biol Chem. 275, 15685-15690 64. Peng, H., Zheng, L., Lee, W. H., Rux, J. J., and Rauscher, F. J., III (2002) Cancer Res. 62, 3773-3781 


\title{
Tetrameric Oligomerization Mediates Transcriptional Repression by the BRCA1-dependent Kruppel-associated Box-Zinc Finger Protein ZBRK1*
}

Received for publication, September 22, 2004, and in revised form, October 5, 2004 Published, JBC Papers in Press, October 20, 2004, DOI 10.1074/jbc.M410926200

\author{
Wei Tan, Seokjoong Kim, and Thomas G. Boyer \\ From the Department of Molecular Medicine and Institute of Biotechnology, University of Texas Health Science \\ Center at San Antonio, San Antonio, Texas 78245-3207
}

The Kruppel-associated box (KRAB)-zinc finger protein ZBRK1 has been implicated in the transcriptional regulation of DNA damage-response genes that function in cell growth control and survival. Recently, we

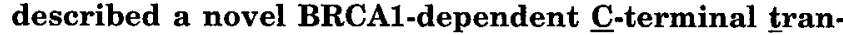
scriptional repression domain (CTRD) within ZBRK1, the mode of repression of which is functionally distinguishable from that of the $N$-terminal KRAB repression domain within ZBRK1. The identification of BRCA1 binding-competent but repression-defective CTRD mutants further revealed that BRCA1 binding is necessary, but not sufficient, for ZBRK1 CTRD function. During an unbiased search for possible co-regulators of the CTRD, we identified ZBRK1 itself, sug. gesting that ZBRK1 can oligomerize through its CTRD. Herein we explore the physical and functional requirements for ZBRK1 oligomerization in ZBRK1-directed transcriptional repression. Protein interaction analyses confirmed that ZBRK1 can homo-oligomerize both in vitro and in vivo and further mapped the ZBRK1 oligomerization domain to the CTRD $\mathrm{C}$ terminus. Biochemical analyses, including protein cross-linking and gel filtration chromatography, revealed that ZBRK1 homo-oligomers exist as tetramers in solution. Functionally, ZBRK1 oligomerization facilitates ZBRK1-directed transcriptional repression through ZBRK1 response elements; requirements for oligomerizationdependent repression include the ZBRK1 CTRD and KRAB repression domains but not the DNA binding activity of ZBRK1. These observations suggest that higher order oligomers of ZBRK1 may assemble on target ZBRK1 response elements through both proteinDNA and CTRD-dependent protein-protein interactions. These findings thus reveal an unanticipated dual function for ZBRK1 in both DNA binding-dependent and -independent modes of transcriptional repression and further establish the CTRD as a novel protein interaction surface responsible for directing homotypic and heterotypic interactions necessary for ZBRK1-directed transcriptional repression.

* This work was supported by United States Army Department of Defense Grant DAMD17-02-1-0584, National Institutes of Health Grant CA098301-01 (to T. G. B.), and United States Army Department of Defense Predoctoral Fellowship DAMD17-03-1-0259 (to W. T.). The costs of publication of this article were defrayed in part by the payment of page charges. This article must therefore be hereby marked "advertisement" in accordance with 18 U.S.C. Section 1734 solely to indicate this fact.

¥ To whom correspondence should be addressed: Dept. of Molecular Medicine and Inst. of Biotechnology, University of Texas Health Science Center at San Antonio, 15355 Lambda Dr., San Antonio, TX 78245-3207. Tel.: 210-567-7258; Fax: 210-567-7377; E-mail: boyer@ uthscsa.edu.

This paper is available on line at http://www.jbc.org
ZBRK1 $^{1}$ (zinc finger and BRCA1-interacting protein with a KRAB domain-1) is a member of the Kruppel-associated boxzinc finger protein (KRAB-ZFP) family of transcriptional repressors $(1,2)$. The $\sim 300$ members of this family comprise a significant proportion of the transcription factor complement of the human proteome and are believed to occupy important regulatory roles in development, differentiation, and transformation (3-9). In addition to its identification as the product of a gene up-regulated in senescent fibroblasts, ZBRK1 was independently identified as a BRCA1-dependent transcriptional repressor of the gene encoding GADD45a, a functionally important DNA damage-response effector that functions in $G_{2} / M$ cell cycle checkpoint control and the maintenance of genomic stability $(2,10,11)$. Previous functional analyses revealed that ZBRK1 represses $G A D D 45 a$ gene transcription through an intron 3 DNA-binding site in a manner dependent upon direct interaction with BRCA1 and further suggested a model in which ZBRK1 and BRCA1 function coordinately to repress GADD45a gene transcription in the absence of genotoxic stress (2). Evidence to support this model derives from the recent observation that DNA damage-induced ubiquitin-mediated proteolysis of ZBRK1 leads to de-repression of $G A D D 45 a$ (12). Although the DNA damage-induced degradation of ZBRK1 is a BRCA1-independent phenomenon, familial breast cancer-derived mutants of BRCA1 that disrupt its interaction with ZBRK1 nonetheless abrogate its co-repressor activity, suggesting that its ZBRK1 co-repressor function may be important for the tumor suppressor properties of BRCA1 $(2,12)$.

In addition to GADD45a, potential ZBRK1 binding sites have been identified in other DNA damage-response genes that are also regulated by $\mathrm{BRCA} 1$, including $p 21, B a x$, and $G A D D 153$ (2). This observation suggests a potentially broader role for BRCA1 and ZBRK1 in the coordinate transcriptional control of functionally diverse DNA damage response genes. As part of our initial effort to explore the mechanism by which BRCA1 mediates sequence-specific transcriptional repression through ZBRK1, we recently identified and functionally characterized a novel $\mathrm{C}$-terminal transcriptional repression domain (CTRD) within ZBRK1 (13). Structurally, the CTRD comprises the last four zinc fingers and an atypical C-terminal extension within the eight-fingered ZBRK1 protein (13). Functionally, the CTRD represses transcription in a BRCA1-dependent, histone deacetylase-dependent, and promoter-specific manner and is thus distinguishable from the $\mathrm{N}$-terminal KRAB repres-

\footnotetext{
1 The abbreviations used are: ZBRK1, zinc finger and BRCA1interacting protein with a KRAB domain-1; BF, broken finger; CTRD, C-terminal transcription repression domain; DSS, disuccinimidyl suberate; KRAB, Kruppel-associated box; MBP, maltose-binding protein; TK, thymidine kinase; ZFP, zinc finger protein; ZRE, ZBRK response element.
} 
sion domain in ZBRK1, which exhibits no BRCA1 dependence and broad promoter specificity (13).

During the course of our functional dissection of ZBRK1, we identified CTRD mutants that are competent for BRCA1 binding but nonetheless defective for transcriptional repression (13). This observation suggests that BRCA1 binding is necessary but not sufficient for the ZBRK1 CTRD repression function and implies a role for additional co-regulators of the CTRD. During an unbiased search for CTRD-interacting proteins and, therefore, potential CTRD co-regulators, we unexpectedly identified ZBRK1 itself, suggesting that ZBRK1 can homo-oligomerize through its CTRD. Herein, we explore the physical and functional requirements for ZBRK1 oligomerization in ZBRK1-directed repression. Our results reveal the ZBRK1 CTRD to be a novel interaction surface responsible for directing both homotypic and the heterotypic interactions essential for ZBRK1 repression and further suggest an unanticipated dual role for ZBRK1 in both DNA binding-dependent and -independent modes of transcriptional repression. We discuss the implications of these findings for BRCA1-dependent ZBRK1 repression.

\section{EXPERIMENTAL PROCEDURES}

Plasmid Construction and Mutagenesis-Plasmid pMAL-C2-TEV ZBRK1 $\triangle \mathrm{K}$ for expressing maltose-binding protein (MBP)-ZBRK1 $\triangle \mathrm{K}$ in Escherichia coli has been described previously (13). pCS2+-FLAGZBRK1 for in vitro transcription/translation and mammalian expression of FLAG epitope-tagged ZBRK1 was constructed by subcloning a BamHI-HindIII fragment carrying sequences encoding an amino-terminally FLAG-tagged ZBRK1 from pFastbac-FLAG-ZBRK1 in to pCS2+ (14). pCS2+-FLAG-ZBRK1 broken finger (BF) and KRAB domain (D12A,V13A) mutants were generated by site-directed mutagenesis using the QuikChange II site-directed mutagenesis kit following the manufacturer's recommendations (Stratagene, La Jolla, CA). Each broken finger mutant bears a histidine (CAT codon) to asparagine (AAT codon) substitution mutation at the first of the two conserved histidine residues within the targeted $\mathrm{C}_{2} \mathrm{H}_{2}$ zinc finger. The D12A,V13A KRAB domain double mutation bears an aspartic acid (GAT codon) to alanine (GGT) substitution and a valine (GTG codon) to alanine (GGG codon) substitution at amino acid position numbers 12 and 13 , respectively, of the 532-amino acid ZBRK1 protein. pCS2+-FLAG-ZBRK1 carboxylterminal truncation mutants $(\triangle \mathrm{C} 1, \Delta \mathrm{C} 2, \Delta \mathrm{C} 3)$ were generated by $\mathrm{PCR}$ based subcloning. In brief, a PstI-XbaI fragment encompassing the carboxyl terminus of ZBRK1 in pCS2+-FLAG-ZBRK1 was replaced with PCR-amplified fragments carrying corresponding carboxyl-terminal truncations. Individual pCS2+-FLAG-ZBRK1 carboxyl-terminal deletion derivatives encode the following ZBRK1 amino acids: $\Delta C 1$, amino acids 1-523; $\Delta \mathrm{C} 2$, amino acids $1-503$; and $\Delta \mathrm{C} 3$, amino acids 1-483. Plasmid pCS2+-T7-ZBRK1 for in vitro transcription/translation and mammalian expression of T7 epitope-tagged ZBRK1 was generated by replacing a BamHI-Ec047-III fragment from pCS2+-FLAG-ZBRK1 with a PCR-amplified BamHI-Eco47-III fragment carrying sequences encoding a T7 epitope tag in-frame with the ZBRK1 amino terminus.

Reporter plasmid pZRE 4 -TK-Luc was constructed by replacing a HindIII-BglII fragment including 5XGAL4 DNA-binding sites and the thymidine kinase (TK) promoter from $\mathrm{pG}_{5}$ TK-Luc (13) with a HindIIIBglII fragment from pBL-CAT E (2), encompassing $4 \times$ ZBRK1 consensus DNA-binding sites and the TK promoter. Plasmid pM-ZBRK1 CTRD (previously named pM-ZBRK1 5ZFC) for mammalian expression of GAL4-CTRD and reporter plasmid $\mathrm{pG}_{5}$ TK-Luc have both been described previously (13).

Cell Culture, Transfections, and Reporter Assays-U2OS human osteosarcoma cells were cultured as described (13). For transient reporter assays, $2 \times 10^{5}$ cells were seeded per well of a 6 -well plate $24 \mathrm{~h}$ before transfection. When $70 \%$ confluent, the cells were transfected with FuGENE 6 (Roche Diagnostics) following the manufacturer's instructions The amounts of expression and reporter plasmids utilized in each experiment are indicated in the legends to Figs. 4-6. A total of 1 or $2 \mu \mathrm{g}$, respectively, of DNA per well was transfected when $\mathrm{pG}_{5}$ TK-Luc or pZRE ${ }_{4}$-Luc were used as reporter templates. Each transfection also included an intermal control plasmid, pCH110 (15), expressing $\beta$-galactosidase under control of the SV40 promoter. Forty-eight hours posttransfection, cells were harvested and lysed in $250 \mu \mathrm{l} /$ well reporter lysis buffer (Promega, Madison, WI). Transfected cell lysates $(20 \mu \mathrm{l})$ were analyzed for luciferase activity using the luciferase assay system (Promega) and for $\beta$-galactosidase activity using the Galacto-Light Plus chemiluminescent reporter assay (BD Biosciences). Each transfection was performed a minimum of three times in duplicate.

Protein Expression and Purification-MBP-ZBRK1 $\triangle \mathrm{K}$, MBPZBRK1 CTRD, and MBP proteins were each expressed in and purified from $E$, coli strain BL21 Star (DE3) pLysS (Invitrogen). The procedures for protein induction, extraction, and purification of MBP-ZBRK1 $\Delta K$ have been described previously (13). For MBP-ZBRK1 CTRD and MBP, cells were grown at $37^{\circ} \mathrm{C}$ to an $A_{600}$ of 0.6 . Isopropyl-1-thio- $\beta$-D-galactopyranoside was added to a final concentration $0.3 \mathrm{~mm}$, and the cells were transferred to $25^{\circ} \mathrm{C}$ for another $3.5 \mathrm{~h}$. Cells were pelleted, washed once with phosphate-buffered saline, and then resuspended in MBP binding buffer (20 mM HEPES, pH 7.5, $1 \mathrm{mM}$ EDTA, $500 \mathrm{mM} \mathrm{NaCl}$, and $10 \mathrm{~mm} \beta$-mercaptoethanol) supplemented with protease inhibitors $(0.4$ $\mu \mathrm{g} / \mathrm{ml}$ aprotinin, $0.5 \mu \mathrm{g} / \mathrm{ml}$ chymostatin, $0.5 \mu \mathrm{g} / \mathrm{ml}$ leupeptin, $0.5 \mu \mathrm{g} / \mathrm{ml}$ pepstatin, $0.5 \mathrm{~mm}$ phenylmethylsulfonyl fluoride, and $0.5 \mathrm{~mm}$ benzamidine-HCl). Resuspended cells were frozen and thawed once followed by sonication (three times for $1 \mathrm{~min}$ each on ice) and clarification by centrifugation at $30,000 \times g$ for $30 \mathrm{~min}$. MBP-ZBRK1 CTRD and MBP proteins were purified from clarified lysates by affinity chromatography on amylose resin (New England Biolabs, Beverly, MA). Briefly, clarified lysates were incubated with amylose resin in batch at for $1 \mathrm{~h}$ at $4{ }^{\circ} \mathrm{C}$, washed with MBP binding buffer, and eluted with MBP binding buffer containing $0.5 \%$ maltose in column format.

$M B P$ Binding Assays-MBP-ZBRK1 $\triangle \mathrm{K}$ was purified from clarified bacterial cell extract $(150 \mu \mathrm{l})$ by incubation with amylose resin $(15 \mu \mathrm{l})$ in batch for $30 \mathrm{~min}$ at $25^{\circ} \mathrm{C}$, followed by washing two times for $5 \mathrm{~min}$ each at $25^{\circ} \mathrm{C}$ in MBP binding buffer supplemented with protease inhibitors and three times for $5 \mathrm{~min}$ each at $25^{\circ} \mathrm{C}$ in lysis 300 buffer $(50 \mathrm{mM}$ Tris-HCl, $300 \mathrm{~mm} \mathrm{NaCl}, 5 \mathrm{~mm}$ EDTA, $0.1 \%$ Nonidet P-40, and $10 \mathrm{~mm}$ $\beta$-mercaptoethanol) supplemented with protease inhibitors. FLAGZBRK1 or its mutant derivatives were labeled with [ ${ }^{35}$ S]methionine (TNT SP6 quick coupled transcription/translation system; Promega) and incubated with amylose-immobilized MBP-ZBRK1 $\Delta \mathrm{K}$ in lysis 300 buffer for $1 \mathrm{~h}$ at $4^{\circ} \mathrm{C}$. Binding reactions were washed with Lysis 300 buffer four times for $10 \mathrm{~min}$ each at $4^{\circ} \mathrm{C}$ and subsequently boiled in $30 \mu \mathrm{l}$ of $1.5 \times$ Laemmli sample buffer. Eluates were resolved by SDS-PAGE (10\% gel) and visualized by PhosphorImager analysis (Amersham Biosciences).

Co-immunoprecipitation Analyses-U2OS cells at $70 \%$ confluency in 10-cm dishes were transfected with $12 \mu \mathrm{g}$ of DNA using the following expression plasmids (with amounts in parentheses): pCS2+-T7-ZBRK1 $(6 \mu \mathrm{g})$ and pCS2+FLAG-ZBRK1 $(6 \mu \mathrm{g}) ; \mathrm{pCS} 2+-\mathrm{T} 7-Z B R K 1(6 \mu \mathrm{g})$ and pCS2+FLAG-ZBRK1 BF mutants (6 $\mu \mathrm{g})$; pCS2+-T7-ZBRK1 $(6 \mu \mathrm{g})$ and pCS2+-FLAG-ZBRK1 $\Delta \mathrm{C} 1(3 \mu \mathrm{g})$, pCS2+-FLAG-ZBRK1 $\Delta \mathrm{C} 2(4.5 \mu \mathrm{g})$, or pCS2+-FLAG-ZBRK1 $\triangle \mathrm{C} 3(2.25 \mu \mathrm{g})$ (the total amount of DNA was fixed by supplementing these transfections with pCS2+). Thirty-six hours post-transfection, $2.5 \times 10^{6}$ transfected U2OS cells were harvested, washed in phosphate-buffered saline, and lysed in $300 \mu 1$ of lysis $150 \mathrm{~T}$ buffer ( $50 \mathrm{~mm}$ Tris-HCl, $\mathrm{pH} 7.5,150 \mathrm{~mm} \mathrm{NaCl}, 5 \mathrm{~mm}$ EDTA, and $0.1 \%$ Nonidet P-40) by sonication at four intervals of $7 \mathrm{~s}$ each using a Branson 450 sonifier (Branson Ultrasonics) at the lowest setting. The lysate was clarified by centrifugation at $20,000 \times g$ for $20 \mathrm{~min}$ at $4^{\circ} \mathrm{C}$. Clarified lysates were subjected to co-immunoprecipitation with either $1 \mu \mathrm{g}$ of anti-T7 antibody (Novagen, Madison, WI) or $5 \mu \mathrm{g}$ of anti-FLAG M2 monoclonal antibody (Sigma-Aldrich) for $4 \mathrm{~h}$ at $4^{\circ} \mathrm{C}$. Immunocomplexes were precipitated with $20 \mu$ of protein A-Sepharose (Amersham Biosciences) (for anti-T7 antibody immunoprecipitation) or protein GSepharose (Roche Diagnostics) (for anti-FLAG antibody immunoprecipitation) for $2 \mathrm{~h}$ at $4{ }^{\circ} \mathrm{C}$. Immunoprecipitates were pelleted and washed with lysis $150 \mathrm{~T}$ buffer at least four times for $10 \mathrm{~min}$ each at $4{ }^{\circ} \mathrm{C}$. Immunoprecipitated proteins were eluted by boiling in $2 \times$ Laemmli sample buffer for $3 \mathrm{~min}$, and eluates were subsequently resolved by SDS-PAGE (10\% gel) and processed for immunoblot analysis.

Chemical Cross-linking Assays-U2OS cells at $70 \%$ confluency on $10-\mathrm{cm}$ plates were transfected with either $12 \mu \mathrm{g}$ of pCS2+-FLAGZBRK1 or $6 \mu \mathrm{g}$ of pCS2+-FLAG-ZBRK1 $\Delta \mathrm{C1}$ in combination with $6 \mu \mathrm{g}$ of pCS2+. Thirty-six hours post-transfection, cells were harvested and lysed in $300 \mu \mathrm{l}$ of lysis $150 \mathrm{H}$ buffer (20 mM HEPES, pH 7.5, $150 \mathrm{~mm}$ $\mathrm{NaCl}, 5 \mathrm{~mm}$ EDTA, and $0.1 \%$ Nonidet $\mathrm{P}-40$ ) by sonication at four intervals of $7 \mathrm{~s}$ each using a Branson 450 sonifier (Branson U/trasonics) at the lowest setting. The lysate was clarified by centrifugation at $20,000 \times g$ for $20 \mathrm{~min}$ at $4^{\circ} \mathrm{C}$. Clarified lysates $(36 \mu \mathrm{l})$ were subjected to chemical cross-linking by the addition of disuccinimidyl suberate (DSS) $(25 \mathrm{~mm})$ to a final concentration of $2.25 \mathrm{~mm}$. Cross-linking reactions were performed at $25^{\circ} \mathrm{C}$ and terminated by the addition of $2 \times$ Laemmli sample buffer followed by boiling for $3 \mathrm{~min}$ (16). Cross-linked products were resolved by SDS-PAGE $(10 \%$ gel) and processed for immunoblot 

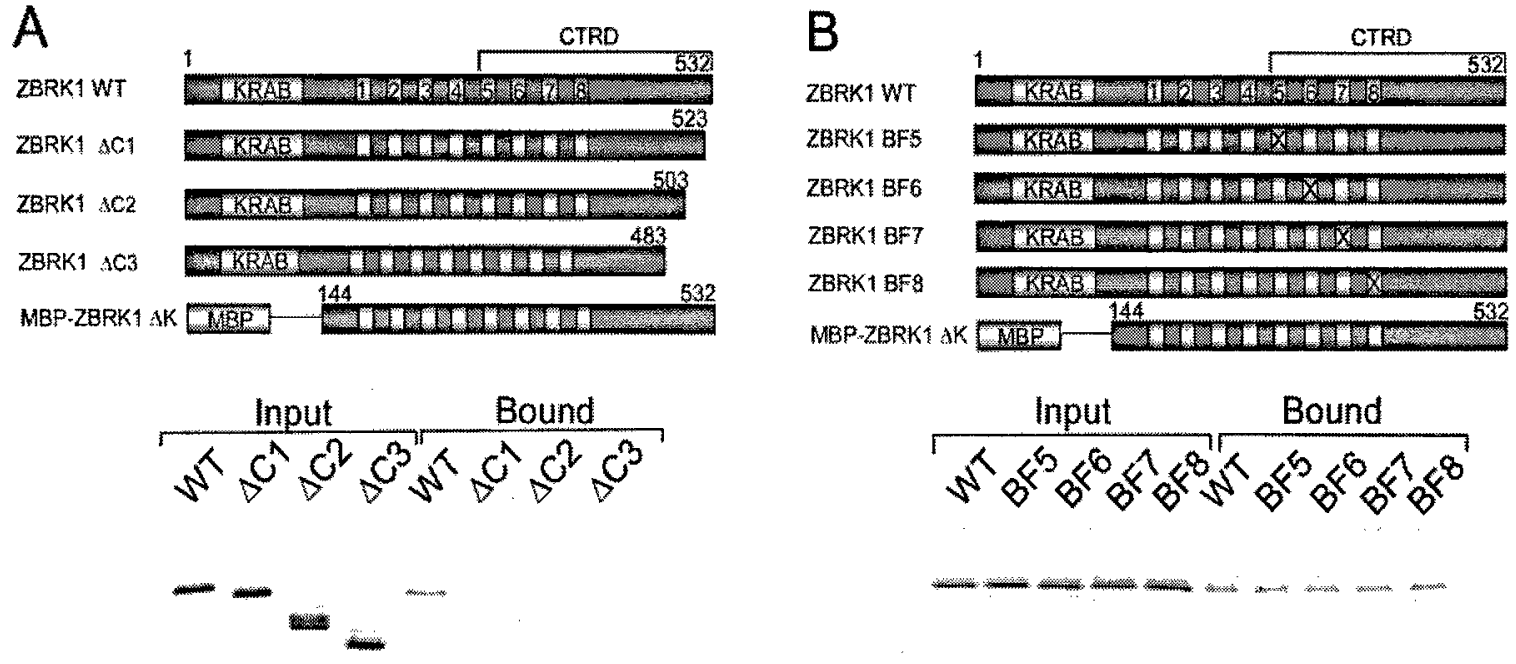

FIG. 1. ZBRK1 homo-oligomerizes in vitro through its CTRD $\mathbf{C}$ terminus. $A$ and $B$, top, schematic representation of FLAG-tagged wild-type $(W T)$ ZBRK1, ZBRK1 carboxyl-terminal deletion $(\triangle C)$ and broken finger $(B F)$ derivatives, and MBP-ZBRK1 $\triangle \mathrm{K}$ (the KRAB and CTRD repression domains as well as numbered zine fingers are indicated). Bottom, purified recombinant MBP-ZBRK1 $\triangle \mathrm{K}$ was immobilized on amylose resin and incubated with in vitro translated ${ }^{35}$ S-labeled wild-type (WT) ZBRK1 (A and $B$ ) or its indicated $\Delta \mathrm{C}(A)$ or BF $(B)$ derivatives. Following extensive washing, bound proteins were eluted in Laemmli sample buffer, resolved by SDS-PAGE (10\% gel), and visualized by PhosphorImager analysis. Input represents $5 \%$ of the total in vitro translated protein used in binding reactions.

analysis using anti-FLAG M2 monoclonal antibody (Sigma-Aldrich).

Purified MBP-ZBRK1 CTRD and MBP proteins were each subjected to cross-linking by the addition of DSS $(25 \mathrm{~mm})$ to a final concentration of $0.75 \mathrm{~mm}$. Cross-linking reactions were performed at $25^{\circ} \mathrm{C}$ and terminated by the addition of $2 \times$ Laemmli sample buffer followed by boiling for $3 \mathrm{~min}$. Cross-linked products were resolved by SDS-PAGE (10\% gel) followed by silver staining.

Gel Filtration Chromatography-U2OS cells at 70\% confluency on 10-cm plates were transfected with either $12 \mu \mathrm{g}$ of pCS2+-FLAGZBRK1 or $6 \mu \mathrm{g}$ of pCS2+-FLAG-ZBRK1 $\Delta \mathrm{C} 1$ in combination with $6 \mu \mathrm{g}$ of pCS2 + . Thirty-six hours post-transfection, cells were harvested and

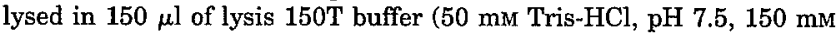
$\mathrm{NaCl}, 5 \mathrm{~mm}$ EDTA, $0.5 \%$ Nonidet P-40, and $25 \mathrm{~mm}$ pentaethylene glycol monooctyl ether (Sigma-Aldrich)) by sonication at four intervals of $7 \mathrm{~s}$ each using a Branson 450 sonifier (Branson Ultrasonics) at the lowest setting. The lysate was clarified by centrifugation at $20,000 \times g$ for 20 min at $4^{\circ} \mathrm{C}$. Clarified lysates were concentrated 5 -fold by centrifugation at 10,000 rpm using a Nanosep 30K omega filtration unit (Pall Corporation, East Hills, NY). Gel filtration chromatography was performed on an Amersham Biosciences SMART System using a Superdex 200 PC $3.2 / 30$ column (Amersham Biosciences) and a flow rate of $40 \mu \mathrm{l} / \mathrm{min}$. Column fractions $(45 \mu \mathrm{l})$ were resolved by SDS-PAGE $(10 \%$ gel) and visualized by immunoblot analysis using an anti-FLAG M2 monoclonal antibody (Sigma-Aldrich).

\section{RESULTS}

ZBRK1 Homo-oligomerizes in Vitro-To identify novel interaction partners and potential co-regulators of the ZBRK1 CTRD, we employed two independent experimental approaches. First, we used the CTRD (amino acids 319-532 of ZBRK1) as a bait protein to screen a human fetal brain cDNA library by a yeast-two hybrid assay. From among $\sim 1 \times 10^{6}$ independent clones screened, we identified ZBRK1 itself as a CTRD interaction partner (data not shown). Second, we expressed a ZBRK1 KRAB domain deletion derivative (ZBRK1 $\Delta \mathrm{K}$; amino acids $144-532$ of ZBRK1) as an MBP chimera in $E$. coli, immobilized the fusion protein on amylose resin, and probed HeLa cell nuclear extract for interacting proteins using an MBP pull-down assay. Identification of ZBRK1 $\Delta \mathrm{K}$-interacting proteins by matrix-assisted laser desorption ionization time-of-flight-based peptide mass fingerprinting revealed ZBRK1 itself to be among the interaction partners thus captured (data not shown). Coupled with the identification of ZBRK1 as a CTRD-interacting protein by a yeast two-hybrid assay, this observation suggested the possibility that ZBRK1 might homo-oligomerize through a direct interaction involving its CTRD.

To confirm that ZBRK1 can bind directly to itself and to map the relevant interaction surface required for this interaction, we tested the ability of in vitro translated wild-type ZBRK1 and ZBRK1 CTRD mutant derivatives for their respective abilities to bind to MBP-ZBRK1 $\triangle \mathrm{K}$ in an MBP pull-down assay (Fig. 1). As reported previously, deletion of the KRAB domain permits the expression and purification of an otherwise insoluble fulllength recombinant ZBRK1 protein (13). MBP-ZBRK1 $\Delta \mathrm{K}$ bound full-length ZBRK1 efficiently, thus confirming that ZBRK1 can directly self-associate in vitro (Fig. $1, A$ and $B$ ). Next, we examined the structural determinants within the CTRD required for ZBRK1 self-association. Analysis of C-terminal ZBRK1 truncation derivatives revealed that the deletion of only nine amino acids from the CTRD $C$ terminus was sufficient to eliminate ZBRK1 oligomerization (Fig. 1A). By contrast, individual disruption of CTRD zinc fingers $5-8$ by substitution mutagenesis revealed that each "broken finger" mutant (13) bound MBP-ZBRK1 $\Delta \mathrm{K}$ comparably to wild-type ZBRK1, thus revealing that the CTRD zinc fingers are individually dispensable for ZBRK1 oligomerization (Fig. 1B). Taken together, these results demonstrate that ZBRK1 can homooligomerize in vitro through a direct interaction involving its CTRD C terminus.

ZBRK1 Homo-oligomerizes in Vivo-To determine whether ZBRK1 can homo-oligomerize in vivo, we first examined the ability of ZBRK1 derivatives independently tagged with either T7 or FLAG epitopes to reciprocally co-immunoprecipitate one another following their transient over-expression in U2OS human osteosarcoma cells. T7-tagged ZBRK1 could be efficiently precipitated by FLAG-specific antibodies only in the presence of FLAG-tagged ZBRK1 (Fig. 2A). Reciprocally, FLAG-tagged ZBRK1 could be efficiently precipitated by T7-specific antibodies only when T7-tagged ZBRK1 was co-expressed (Fig. $2 B$ ). These results confirm that ZBRK1 can homo-oligomerize in vivo. Next, to map the relevant interaction surface(s) required for ZBRK1 oligomerization in vivo, we examined a set of FLAGtagged ZBRK1 CTRD mutant derivatives for their respective 


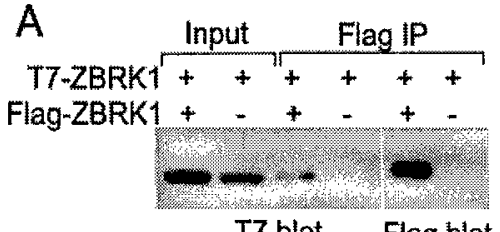

T7 blot

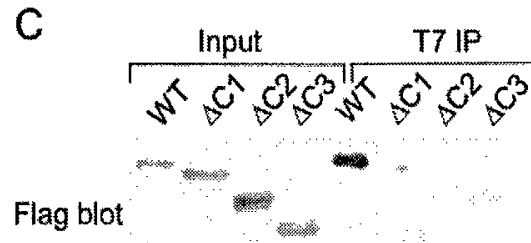

T7 blot
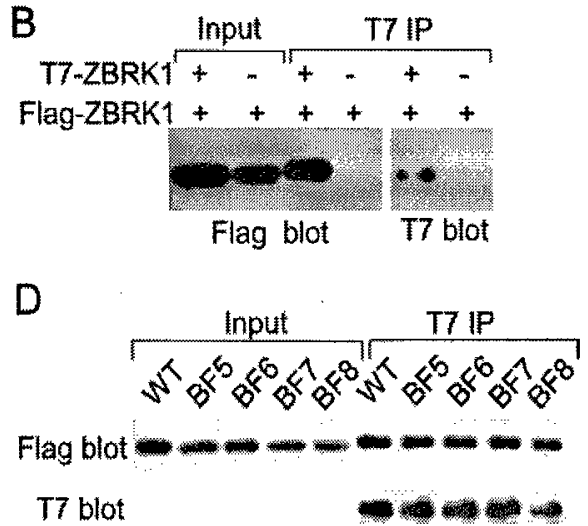

FIG. 2. ZBRK1 homo-oligomerizes in vivo through its CTRD $\mathbf{C}$ terminus. $A$ and $B$, T7 and FLAG-tagged wild-type ZBRK1 proteins were ectopically expressed either alone or together in U20S cells, and whole cell extracts from transfected cells were subjected to immunoprecipitation $(I P)$ using antibodies specific for either the FLAG $(A)$ or T7 $(B)$ epitopes. Immunoprecipitates were resolved by SDS-PAGE (10\% gel) and processed by immunoblot analysis using both T7 and FLAG epitope-specific antibodies as indicated. Input represents $10 \%$ of the total whole cell extract subjected to immunoprecipitation. $C$ and $D$, T7-tagged wild-type (WT) ZBRK1 was ectopically expressed in U20S cells along with FLAG-tagged WT ZBRK1 or its indicated $\triangle \mathrm{C}(C)$ or $\mathrm{BF}(D)$ derivatives. Whole cell extracts from transfected cells were subjected to immunoprecipitation $(I P)$ using antibodies specific for the T7 epitope. Immunoprecipitates were resolved by SDS-PAGE (10\% gel) and processed by immunoblot analysis using both T7- and FLAG epitope-specific antibodies as indicated. Input represents $10 \%$ of the total whole cell extract subjected to immunoprecipitation.

abilities to be co-immunoprecipitated along with T7-tagged wild-type ZBRK1 following their transient co-expression in U2OS cells. Consistent with the results of in vitro binding analyses, these in vivo binding studies revealed the CTRD C terminus to be critical and the CTRD zinc fingers to be dispensable for ZBRK1 self-association. Thus, deletion of only nine amino acids from the ZBRK1 $\mathrm{C}$ terminus largely abolished ZBRK1 self-association (Fig. 2C), whereas individual ZBRK1 derivatives bearing targeted disruptions of CTRD zinc fingers 5-8 bound to T7-tagged ZBRK1 as efficiently as wild-type ZBRK1 (Fig. 2D). Taken together, these results demonstrate that ZBRK1 can homo-oligomerize both in vitro and in vivo and furthermore delimit the oligomerization interface to the CTRD $\mathrm{C}$ terminus.

The ZBRK1 CTRD Mediates Tetrameric Oligomerization-To characterize the oligomeric state of ZBRK1 in vivo, we first employed the chemical cross-linker DSS to covalently capture ZBRK1 protein complexes present in extracts of U2OS cells transfected with FLAG-tagged ZBRK1 (16). Cross-linked samples were analyzed by SDS-PAGE and immunoblot analysis using FLAG epitope tag-specific antibodies. In the presence of DSS, a significant proportion of FLAG-tagged ZBRK1 migrated with an apparent molecular mass of $\sim 240 \mathrm{kDa}$, consistent with the size of a ZBRK1 tetramer (Fig. 3A). Importantly, no cross-linked products were observed when an oligomerization-defective ZBRK1 mutant $(\triangle \mathrm{C} 1)$ was over-expressed in U2OS cells, demonstrating that DSS-induced formation of high molecular mass ZBRK1 complexes is strictly dependent upon the intact CTRD C terminus (Fig. 3A).

Although the electrophoretic properties of FLAG-tagged ZBRK1 in DSS-cross-linked U2OS cell extracts suggests the formation of tetramers, we could not exclude the possibility that additional cellular proteins were also incorporated into the ZBRK1 complex, particularly because we know that ZBRK1 interacts with other proteins in the cell, including BRCA1 (2, 13). The concentration of ectopically expressed ZBRK1 in these transfection experiments considerably exceeds that of its endogenous counterpart, and these conditions are likely to drive ZBRK1 self-association and mask the influence of endogenous interacting proteins on the size of a cross-linked ZBRK1 complex. Nonetheless, we sought independent confirmation of the stoichiometry of ZBRK1 oligomerization through its CTRD. To this end, we therefore subjected highly purified recombinant MBP-ZBRK1 CTRD to DSS cross-linking. Cross-linked samples were analyzed by SDS-PAGE followed by silver staining to visualize MBP-ZBRK1 CTRD oligomers. In the presence of DSS, a significant proportion of MBP-ZBRK1 CTRD migrated with an apparent molecular mass of $\sim 270 \mathrm{kDa}$, consistent with the size of an MBP-ZBRK1 CTRD tetramer (Fig. $3 B$ ). Importantly, no cross-linked products were observed when MBP alone was treated with DSS, indicating that DSS-induced formation of a high molecular mass MBP-ZBRK1 CTRD complex is strictly dependent upon the ZBRK1 CTRD (Fig. 3B). Furthermore, immunoblot analysis using ZBRK1-specific antibodies confirmed that the high molecular mass complex observed upon DSS cross-linking of highly purified MBP-ZBRK1 CTRD is derived from MBP-CTRD oligomerization (data not shown).

As an independent approach to determine the stoichiometry of ZBRK1 oligomerization through its CTRD, we examined the respective gel filtration profiles of ectopically expressed wildtype ZBRK1 and its oligomerization-defective mutant $(\Delta \mathrm{C1})$ present in extracts of transfected U2OS cells. Immunoblot analysis of column fractions using a FLAG epitope-specific antibody revealed a peak of wild-type ZBRK1 protein in fractions corresponding to an apparent molecular mass of $\sim 240$ $\mathrm{kDa}$, most consistent with the size of a ZBRK1 tetramer (Fig. $3 C$ ). We also noted a minor peak of wild-type ZBRK1 protein in a fraction corresponding to an apparent ZBRK1 monomer of 58 $\mathrm{kDa}$. By contrast, an oligomerization-defective ZBRK1 derivative ( $\triangle \mathrm{C} 1)$ peaked exclusively in column fractions corresponding to an apparent monomer of $\sim 57 \mathrm{kDa}$ (Fig. $3 C$ ). Taken together, these results indicate that ZBRK1 tetramerizes both in vitro and in vivo in a manner that is strictly dependent upon the integrity of its CTRD C terminus.

ZBRK1 Homo-oligomerization Potentiates ZBRK1-directed Transcriptional Repression-To explore the functional consequence of ZBRK1 homo-oligomerization, we initially exploited our previous observation that the ZBRK1 CTRD can function as an autonomous transcriptional repression domain when tethered to a heterologous DNA-binding domain from the yeast transcription factor GAL4 (13). First, we examined the ability of full-length ZBRK1 to potentiate transcriptional repression 


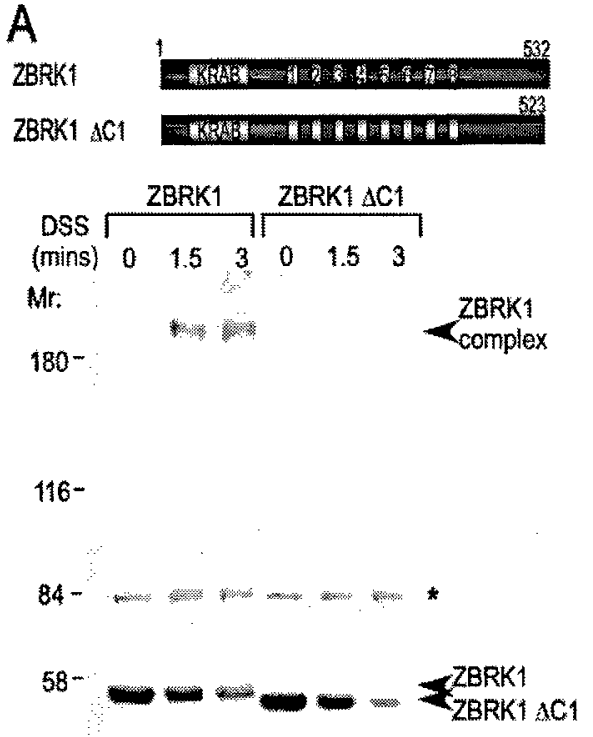

Flag blot

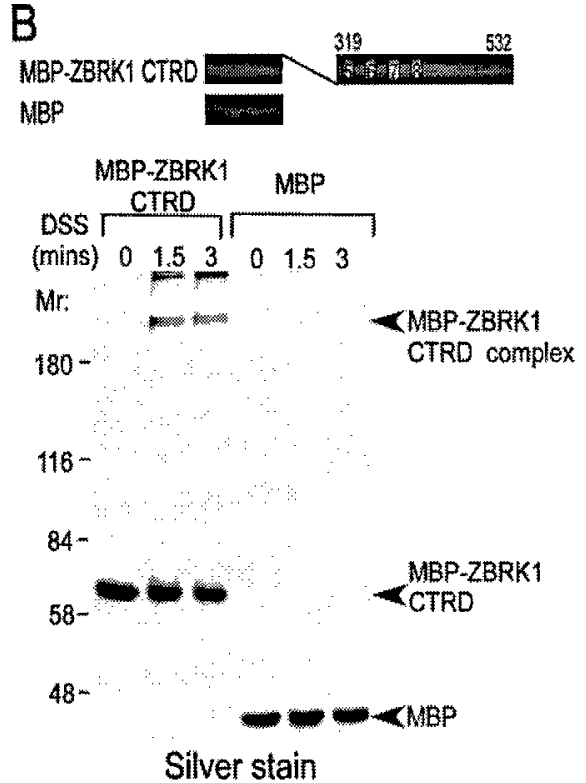

Silver stain

C

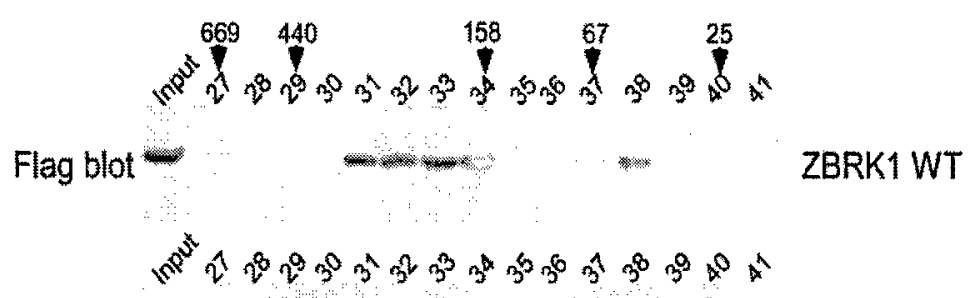

Flag blot

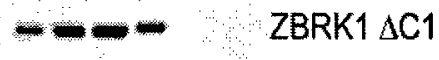

FIG. 3. ZBRK1 tetramerization requires its CTRD C terminus. A, schematic representation of FLAG-tagged wild-type ZBRK1 and its oligomerization-defective $\Delta \mathrm{C} 1$ deletion derivative. U2OS cells were transfected with expression vectors for either FLAG-tagged WT ZBRK1 or its $\Delta \mathrm{C} 1$ deletion derivative as indicated. Whole cell extracts from transfected cells were subjected to cross-linking with $2.25 \mathrm{mM}$ DSS for $0,1.5$, or 3 min as indicated, and cross-linking reactions were terminated with Laemmli sample buffer. Cross-linked proteins were resolved by SDS-PAGE $(10 \%$ gel) and visualized by immunoblot analysis using FLAG epitope-specific antibodies. Arrowheads indicate un-cross-linked monomers of FLAG-tagged ZBRK1 and its $\triangle \mathrm{C} 1$ deletion derivative as well as cross-linked ZBRK1 complexes. Molecular weight markers $\left(M_{\mathrm{r}}\right)$ are indicated. Asterisk denotes a cross-reacting cellular protein recognized by the FLAG antibody. $B$, schematic representation of recombinant MBP-ZBRK1 CTRD and MBP. Purified recombinant MBP-ZBRK1 CTRD and MBP were subjected to cross-linking with $0.75 \mathrm{mM}$ DSS for $0,1.5$, or $3 \mathrm{~min}$ as indicated, and cross-linking reactions were terminated with Laemmli sample buffer. Cross-linked proteins were resolved by SDS-PAGE (10\% gel) and visualized by silver stain analysis. Arrowheads indicate un-cross-linked monomers of MBP-ZBRK1 CTRD and MBP as well as cross-linked MBP-ZBRK1 CTRD complexes. Molecular weight markers $\left(M_{\mathrm{r}}\right)$ are indicated. $C$, whole cell extracts from U2OS cells transfected with FLAG-tagged wild-type (WT) ZBRK1 or an oligomerization-defective mutant derivative ( $\triangle \mathrm{C1}$ ) were independently subjected to Superdex 200 gel filtration chromatography. Individual column fractions were resolved by SDS-PAGE (10\% gel) and analyzed for the presence of FLAG-tagged ZBRK1 by immunoblot analysis with FLAG epitope-specific antibodies. Arrowheads indicate the relative positions of marker protein peaks. Input represents $5 \%$ of the total whole cell extract subject to gel filtration chromatography.

directed by GAL4-ZBRK1 CTRD in a transient repression assay using a reporter template bearing multimerized GAL4 DNA-binding sites located upstream of the herpes simplex virus TK promoter in U2OS cells (Fig. $4 A$ ) (13). Consistent with our previous studies (13), we observed that GAL4-ZBRK1 CTRD repressed transcription from this reporter template at least 10 -fold in a dose-dependent manner (data not shown). To examine the influence of wild-type ZBRK1 on repression directed by GAL4-ZBRK1 CTRD, we transfected a sub-optimal nanogram quantity of the GAL4-ZBRK1 CTRD expression vector that supports only $\sim 2.5$-fold repression of reporter gene activity. Under these conditions, ectopic expression of wild-type ZBRK1 enhanced this level of repression up to 4 -fold in a dose-dependent manner (Fig. $4 B$ ). Importantly, ZBRK1 had minimal influence on reporter activity in the presence of the GAL4 DNA-binding domain alone, confirming that the repressive effect of ZBRK1 in this assay derives from its direct recruitment to the CTRD and not to DNA (Fig. $4 B$ ). This result demonstrates for the first time that, in addition to its well documented role as a sequence-specific DNA-binding transcriptional repressor $(2,13)$, ZBRK1 can also function as a corepressor independent of its inherent DNA binding activity.

Next, we examined the structural features within ZBRK1 required for its co-repressor function. Relative to wild-type ZBRK1, oligomerization-defective CTRD C-terminal truncation mutants ( $\Delta \mathrm{C} 1, \Delta \mathrm{C} 2$, and $\Delta \mathrm{C} 3)$ were completely defective for ZBRK1 co-repressor activity, implying a strict reliance on oligomerization for ZBRK1 to function as a co-repressor (Fig. $5 A$ ). Analysis of ZBRK1 derivatives bearing targeted disruptions of CTRD zinc fingers 5-8 (BF 5-8) revealed these mutants to be partially defective for the ZBRK1 co-repressor function (Fig. $5 B$ ). Because these CTRD zinc finger mutations do not disrupt ZBRK1 oligomerization (see Figs. 1 and 2) but do disrupt CTRD interactions with additional co-repressors including BRCA1 (13), this result suggests that CTRD-mediated recruitment of additional co-repressors also likely contributes to the ability of ZBRK1 to function as a DNA binding-independent co-repressor. Finally, we examined the contribution of the 
A

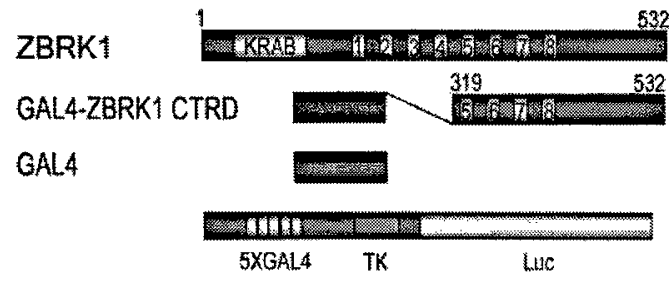

B

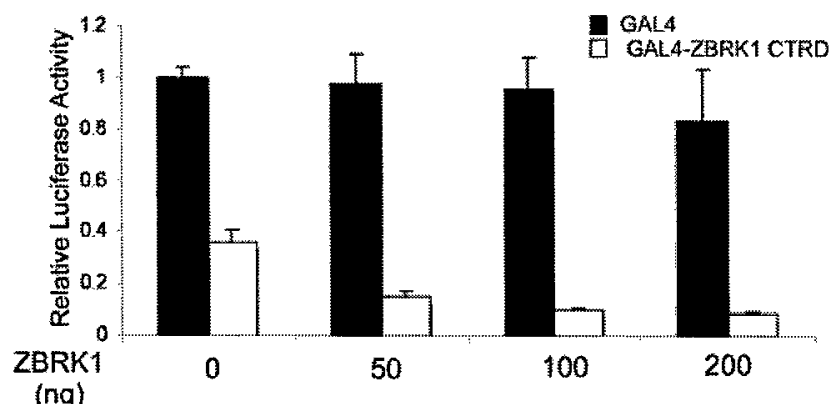

FIG. 4. ZBRK1 has a DNA binding-independent co-repressor activity. $A$, schematic representation of FLAG-tagged ZBRK1, GAL4ZBRK1-CTRD, GAL4 DNA-binding domain alone, and the $\mathrm{pG}_{5} \mathrm{TK}$-lucerferase (5XGAL4 TK Luc) reporter template used in transfection assays. $B$, U2OS cells were transfected with $100 \mathrm{ng}$ of the $\mathrm{pG}_{5} \mathrm{TK}$. luciferase reporter template and $600 \mathrm{ng}$ of either $\mathrm{pM}$ (expressing the GAL4 DNA-binding domain alone) or pM-ZBRK1-CTRD (expressing GAL4-ZBRK1-CTRD) without or with the indicated nanogram amounts of pCS2+-FLAG-ZBRK1 expressing FLAG-tagged wild-type ZBRK1. In this and all subsequent transient reporter assays involving effector plasmid titrations, the total amount of DNA in each transfection was fixed by reciprocal titration of the corresponding backbone expression plasmid. Also, in this and all subsequent transient reporter assays the relative luciferase activity represents the ratio of the luciferase activity obtained in a particular transfection to that obtained in cells transfected with only the reporter and pM expression vectors alone. Luciferase activities were first normalized to $\beta$-galactosidase activity obtained by co-transfection of the SV40- $\beta$-galactosidase vector ( $25 \mathrm{ng}$ ) as described previously (13). Error bars represent the S.D. from the average of at least three independent transfections performed in duplicate.

$\mathrm{N}$-terminal KRAB repression domain within ZBRK1 to its corepressor activity. Relative to wild-type ZBRK1, a ZBRK1 mutant derivative bearing two substitution mutations $(D V \rightarrow A A)$ within the KRAB domain previously shown to disrupt KRAB repression function (17) was significantly defective for co-repressor activity (Fig. 5C). As this mutation does not affect ZBRK1 oligomerization, this result suggests that KRAB domain-associated co-repressors also contribute to the ability of ZBRK1 to function as a DNA binding-independent co-repressor of the CTRD. Taken together these results reveal that ZBRK1, in a DNA-binding-independent manner, can potentiate the repression function of DNA-bound CTRD through oligomerization and the consequent recruitment of co-repressors through both its KRAB and CTRD repression domains.

We next asked whether ZBRK1 CTRD-mediated oligomerization contributes to ZBRK1-directed transcriptional repression from ZBRK1 response elements (ZREs). To this end, we employed a reciprocal transient repression assay in which we examined the ability of the CTRD to potentiate ZBRK1-directed repression from a reporter template bearing multimerized ZREs (Fig. 6A). The fact that the CTRD by itself has no ZRE binding activity permitted us to assess the influence of the CTRD on ZBRK1-directed repression solely by virtue of its ability to oligomerize with DNA-bound ZBRK1. Consistent with our previous studies (2), we observed that wild-type ZBRK1 repressed transcription from a reporter template bearing multimerized ZREs located upstream of the HSV TK pro- moter in U2OS cells at least 10-fold in a dose-dependent manner (data not shown). To examine the influence of the ZBRK1 CTRD on repression directed by wild-type ZBRK1, we transfected a sub-optimal nanogram quantity of the ZBRK1 expression vector that supports only $\sim 2.5$-fold repression of transcription from this reporter template (Fig. 6B). Under these conditions, ectopic expression of GAL4-CTRD enhanced this level of repression up to 4 -fold in a dose-dependent manner (Fig. 6B). Importantly, GAL4-CTRD had minimal influence on reporter activity in the absence of ZBRK1, confirming that the CTRD potentiates ZBRK1 repression in this assay by virtue of its direct recruitment to ZBRK1 and not to DNA (Fig. 6B). Furthermore, an oligomerization-defective CTRD mutant (GAL4-CTRD $\triangle \mathrm{C1}$ ) bearing a nine amino acid deletion from the CTRD C terminus was completely defective in its ability to potentiate ZBRK1 repression in this assay, confirming that CTRD-mediated oligomerization is a requirement for its ZBRK1 co-repressor activity (Fig. 6C). Collectively, these experiments reveal an unanticipated dual function for ZBRK1 in both DNA binding-dependent and -independent modes of repression, the latter of which derives from the ability of ZBRK1 to homo-oligomerize through its CTRD.

\section{DISCUSSION}

The identification of ZBRK1 itself during an unbiased search for ZBRK1 CTRD co-regulators prompted us to characterize the structural requirements for and the functional consequences of ZBRK1 homo-oligomerization. Herein we demonstrate that ZBRK1 undergoes tetrameric oligomerization both in vitro and in vivo, and we further identify the ZBRK1 CTRD C terminus to be both necessary and sufficient for tetrameric oligomerization. Functionally, we demonstrate that ZBRK1 oligomerization facilitates ZBRK1-directed transcriptional repression and concomitantly identify several unique and biologically significant features of ZBRK1 relevant to its role as a KRAB-ZFP transcriptional repressor. First, by virtue of its ability to homooligomerize, we identify an unanticipated role for ZBRK1 as a DNA binding-independent co-repressor in addition to its well characterized role as a sequence-specific DNA-binding transcriptional repressor (2). In this regard, we identify both the $\mathrm{KRAB}$ and CTRD repression domains within ZBRK1 to be necessary for oligomerization-dependent repression. Whether or not these domains are sufficient for oligomerization-dependent repression remains to be established. Second, we reveal the ZBRK1 CTRD to be a novel protein interaction surface responsible for directing both homotypic and the heterotypic interactions necessary for BRCA1-dependent ZBRK1 transcriptional repression.

In general, oligomerization enhances regulatory diversity among sequence-specific DNA-binding transcription factors by expanding the number of potential response elements to which oligomers can effectively bind and/or by increasing the number and type of transcriptional regulatory domains that can effectively function at a core promoter. Presently, we do not know whether and how ZBRK1 oligomerization might influence the choice of DNA sequences recognized by ZBRK1. The 15-base pair consensus ZRE was originally identified by sampling a random pool of double-stranded oligonucleotides with a recombinant ZBRK1 derivative comprised solely of the eight ZBRK1 zinc fingers but lacking the C-terminal extension that we show here to be critical for oligomerization (2). Future studies will be required to determine whether and how oligomerization-competent forms of ZBRK1 preferentially recognize an expanded ZRE. Interestingly, our most recent studies revealed that the inherent DNA binding activity of ZBRK1 zinc fingers 1-7 on a consensus ZRE is constrained by the ZBRK1 $\mathrm{C}$ terminus, suggesting a potential role for the ZBRK1 oligomerization domain in regulating the 

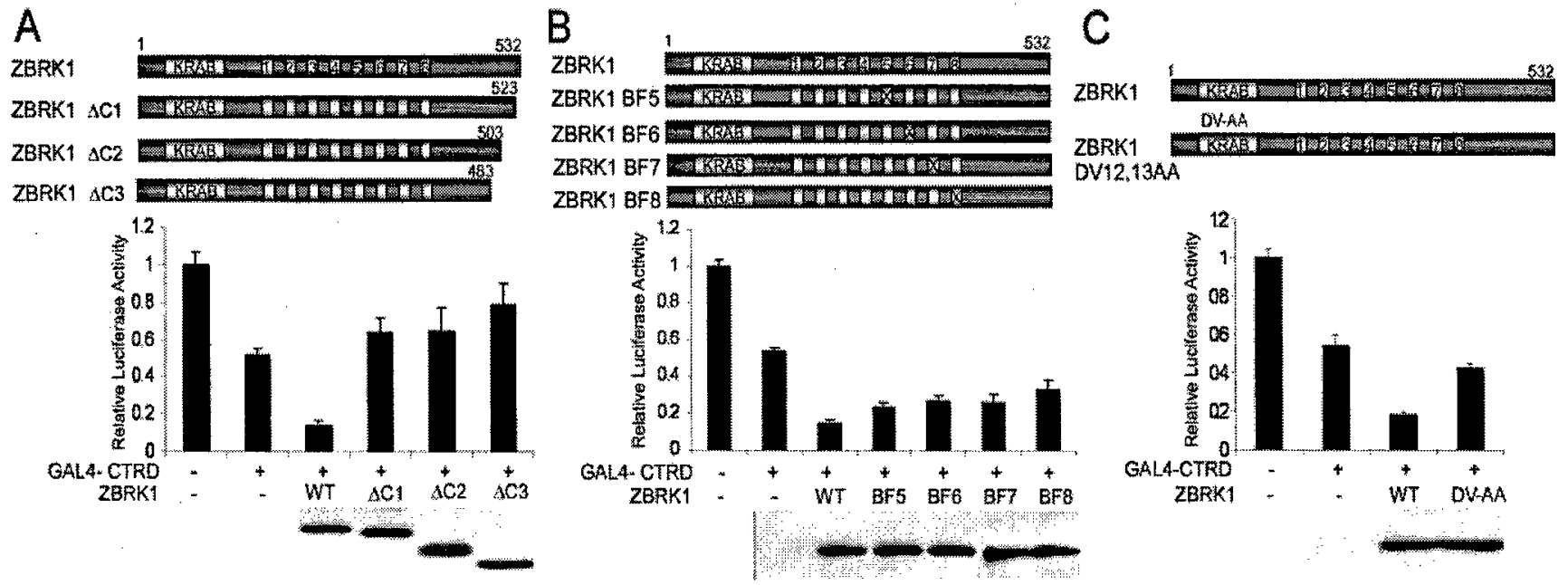

FIG. 5. Structural requirements for ZBRK1 co-repressor activity. $A-C$, top, schematic representation of FLAG-tagged wild-type ZBRK1 and its $\triangle \mathrm{C}(A), \mathrm{BF}(B)$, and $\mathrm{KRAB}$ domain $(C)$ mutant derivatives. Middle, U20S cells were transfected with $100 \mathrm{ng}$ of the pG $\mathrm{g}_{5}$ TK-luciferase reporter template without or with $600 \mathrm{ng}$ of pM-ZBRK1-CTRD expressing GAL4-ZBRK1-CTRD as indicated and the normalized amounts of pCS2+-FLAGZBRK1 expressing FLAG-tagged ZBRK1 or its indicated mutant derivatives (200 ng each of wild-type ZBRK1 (WT), ZBRK1 BF derivatives, and ZBRK1 D12A,V13A $(D V-A A) ; 100 \mathrm{ng}$ of ZBRK1 $\Delta \mathrm{C} 1 ; 150 \mathrm{ng}$ of $\Delta \mathrm{C} 2$; and $75 \mathrm{ng}$ of $\Delta \mathrm{C} 3)$. Relative luciferase activities were calculated as described in the legend to Fig. 4. Bottom, to confirm that wild-type FLAG-tagged ZBRK1 and its mutant derivatives were expressed at roughly equivalent levels in functional assays, equivalent proportional aliquots of transfected U20S cell lysates used in functional assays (middle) were also resolved by SDS-10\% PAGE and subjected to immunoblot analysis using FLAG epitope-specific antibodies.
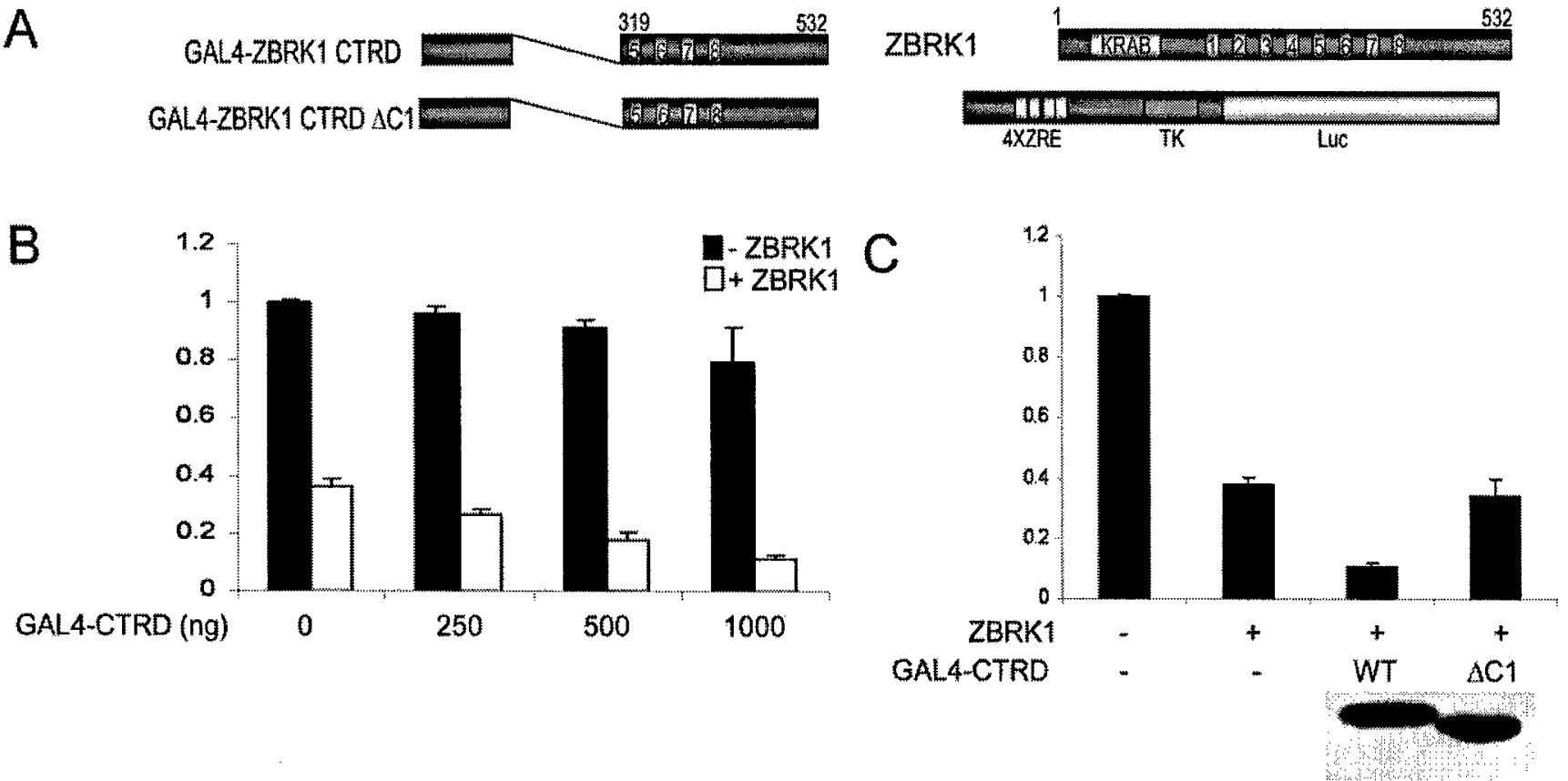

FIG. 6. CTRD-mediated oligomerization is essential for ZBRK1 co-repressor activity. A, schematic representation of GAL4-ZBRK1CTRD, GAL4-ZBRK1-CTRD $\triangle \mathrm{C1}$, FLAG-tagged ZBRK1, and a pZRE TK$_{4}$-luciferase reporter (4XZRE TK Luc) template used in transfection assays. $B$, U20S cells were transfected with $100 \mathrm{ng}$ of the pZRE $\mathrm{TK}$-Luc reporter template without or with $800 \mathrm{ng}$ of pCS2+-FLAG-ZBRK1 expressing FLAG-tagged ZBRK1 and the indicated nanogram amounts of pM-ZBRK1 CTRD expressing GAL4-ZBRK1 CTRD. $C$, U2OS cells were transfected with $100 \mathrm{ng}$ of the pZRE ${ }_{4}$ TK-Luc reporter template without or with $800 \mathrm{ng}$ of pCS2+-FLAG-ZBRK1 expressing FLAG-tagged ZBRK1 and normalized amounts of pM-based plasmids expressing GAL4-ZBRK1 CTRD (1000 ng) or GAL4-ZBRK1-CTRD $\triangle \mathrm{C1}$ (500 ng). To confirm that wild-type (WT) GAL4-ZBRK1 CTRD and GAL4-ZBRK1-CTRD $\triangle C 1$ were expressed at equivalent levels in functional assays, equivalent proportional aliquots of transfected U20S cell lysates used in the functional assays were also resolved by SDS-PAGE (10\% gel) and subjected to immunoblot analysis using FLAG epitope-specific antibodies.

sequence-specific association of ZBRK1 with a consensus ZRE (13). Although our previous studies clearly revealed that ZBRK1 can bind to and repress transcription from a single ZRE $(2,13)$, it nonetheless remains to be established whether and how ZBRK1 oligomerization might facilitate synergistic binding and/or repression from multimerized ZREs.

Our work reveals a unique and unanticipated dual function for ZBRK1 in both DNA binding-dependent and -independent modes of transcriptional repression. With respect to the latter, we demonstrate that ZBRK1 can function as a co-repressor in a manner dependent upon the integrity of its CTRD and KRAB repression domains but not upon its inherent DNA binding activity. This observation raises the possibility that the regulatory potential of ZBRK1 on certain target genes may derive from its recruitment via protein-protein rather than proteinDNA interactions. To our knowledge, this represents the first 
example of a KRAB-ZFP that can function dually as both a DNA-binding repressor as well as a DNA binding-independent co-repressor. Thus, we propose that higher order oligomers of ZBRK1 may assemble on a target ZRE through protein-DNA and protein-protein interactions, the latter involving oligomerization through the CTRD C terminus.

Our work further provides unique insight into the structural and functional organization of ZBRK1 as a member of the KRAB-ZFP family. Like other KRAB-ZFPs, ZBRK1 harbors an $\mathrm{N}$-terminal KRAB repression domain followed by a $\mathrm{C}_{2} \mathrm{H}_{2}$ zinc finger DNA-binding domain. However, ZBRK1 also harbors an atypical C-terminal extension that is generally absent from the larger family of KRAB-ZFPs. Our previous work revealed this C-terminal extension to comprise part of a novel BRCA1-dependent CTRD that also includes zinc fingers 5-8 within ZBRK1 (13). Herein, we describe an additional and unique role for the CTRD as an oligomerization interface sufficient for directing homotypic interactions critical for ZBRK1 repressor function. Within the CTRD we demonstrate that zinc fingers 5-8 are dispensable, whereas the C-terminal extension is essential for ZBRK1 oligomerization. This C-terminal extension shows no obvious sequence similarity to the SCAN domain that mediates selective oligomerization between certain KRABZFPs $(1,18)$. However, a BLAST search of available protein databases reveals several additional KRAB-ZFPs (ZNF577, ZNF613, and FLJ12644), each of which carries an extended C terminus of variable length with sequence homology to the ZBRK1 C-terminal extension. Interestingly, these proteins exhibit the same rank order with respect to their degree of homology and physical proximity to ZBRK1 on chromosome 19q, suggesting that they may have arisen from gene duplication (19-21). It will be of future interest to determine whether and how these KRAB-ZFPs functionally oligomerize with ZBRK1 and/or BRCA1. Although zinc fingers 5-8 within the CTRD are dispensable for oligomerization, our previous studies have nonetheless shown them to be essential for mediating interactions with additional co-repressors, including BRCA1 $(2,13)$. Thus, our studies collectively reveal the CTRD to be a novel protein-interaction surface responsible for directing both homotypic and heterotypic interactions necessary for BRCA1dependent ZBRK1 transcriptional repression.

Finally, our identification of a novel oligomerization domain within ZBRK1 suggests that the regulatory potential of ZBRK1 could extend to genes that do not contain ZREs. For example, ZBRK1 could repress transcription through its hetero-oligomerization with other sequence-specific DNA binding transcription factors through direct protein-protein interactions involving its $\mathrm{C}$ terminus. This, in turn, could expand the repertoire of target genes subject to coordinate transcriptional control by both ZBRK1 and BRCA1, because the repressive potential of ZBRK1 is dependent upon BRCA1 $(2,13)$. Consistent with this possibility, our yeast two-hybrid screen for CTRDinteracting proteins identified, in addition to ZBRK1 itself, the oligomeric transcription factors SRF and ATF-1 (22-26). Interestingly, these transcription factors control the expression of genes with diverse functions in cell growth control and survival, and future studies will seek to establish whether and how ZBRK1 physically and functionally interacts with these transcriptional regulators. Nonetheless, our work here sheds new light on the structural and functional organization of ZBRK1 as a KRAB-ZFP and further defines the multifunctional nature of its unique CTRD.

Acknowledgements-We thank our laboratory colleagues for advice, discussion, and comments.

\section{REFERENCES}

1. Collins, T., Stone, J. R., and Williams, A. J. (2001) Mol. Cell. Biol. 21, $3609-3615$

2. Zheng, L., Pan, H., Li, S., Flesken-Nikitin, A., Chen, P. L., Boyer, T. G., and Lee, W. H. (2000) Mol. Cell 6, 757-768

3. Bellefroid, E. J., Marine, J. C., Ried, T., Lecocq, P. J., Riviere, M., Amemiya, C., Poncelet, D. A., Coulie, P. G., de Jong, P., Szpirer, C., Ward, D. C., and Martial, J. A. (1993) EMBO J. 12, 1363-1374

4. Bellefroid, E. J., Poncelet, D. A., Lecocq, P. J., Revelant, O., and Martial, J. A. (1991) Proc. Natl. Acad. Sci. U. S. A. 88, 3608-3612

5. Theon, A. H., Ganss, B., Cheifetz, S., and Sodek, J. (2001) J. Biol. Chem. 276, 18282-18289

6. Krebs, C. J., Larkins, L. K., Price, R., Tullis, K. M., Miller, R. D., and Robins, D. M. (2003) Genes Dev. 17, 2664-2674

7. Gebelein, B., Fernandez-Zapico, M., Imoto, M., and Urrutia, R. (1998) J. Clin. Investig. 102, 1911-1919

8. Gebelein, B., and Urrutia, R. (2001) Mol. Cell. Biol. 21, 928-939

9. Wagner, S., Hess, M. A., Ormonde-Hanson, P., Malandro, J., Hu, H., Chen, M., Kehrer, R., Frodsham, M., Schumacher, C., Beluch, M., Honer, C., Skolnick, M., Ballinger, D., and Bowen, B. R. (2000) J. Biol. Chem. 275, 15685-15690

10. Sheikh, M. S., Hollander, M. C., and Fornance, A. J., Jr. (2000) Biochem. Pharmacol. 59, 43-45

11. Ran, Q., Wadhwa, R., Bischof, O., Venable, S., Smith, J. R., and Pereira-Smith, O. M. (2001) Exp. Cell Res. 263, 156-162

12. Yun, J., and Lee, W. H. (2003) Mol. Cell. Biol. 23, 7305-7314

13. Tan, W., Zheng, L., Lee, W. H., and Boyer, T. G. (2004) J. Biol. Chem. 279, $6576-6587$

14. Turner, D. L., and Weintraub, H. (1994) Genes Dev. 8, 1434-1447

15. Zheng, L., Annab, L. A., Afshari, C. A., Lee, W. H., and Boyer, T. G. (2001) Proc. Natl. Acad. Sci. U. S. A. 98, 9587-9592

16. McCarty, A. S., Kleiger, G., Eisenberg, D., and Smale, S. T. (2003) Mol. Cell 11, $459-470$

17. Margolin, J. F., Friedman, J. R., Meyer, W. K., Vissing, H., Thiesen, H. J., and Rauscher, F. J., III (1994) Proc. Natl. Acad. Sci. U. S. A. 91, 4509-4513

18. Schumacher, C., Wang, H., Honer, C., Ding, W., Koehn, J., Lawrence, Q., Coulis, C. M., Wang, L. L., Ballinger, D., Bowen, B. R., and Wagner, S. (2000) J. Biol. Chem. 275, 17173-17179

19. Shannon, M., Ashworth, L. K., Mucenski, M. L., Lamerdin, J. E., Branscomb, E., and Stubbs, L. (1996) Genomics 33, 112-120

20. Shannon, M., Kim, J., Ashworth, L., Branscomb, E., and Stubbs, L. (1998) DNA Seq. 8, 303-315

21. Shannon, M., Hamilton, A. T., Gordon, L., Branscomb, E., and Stubbs, L. (2003) Genome Res. 13, 1097-1110

22. Mayr, B., and Montminy, M. (2001) Nat. Rev. Mol. Cell Biol. 2, 599-609

23. De Cesare, D., and Sassone-Corsi, P. (2000) Prog. Nucleic Acids Res. Mol. Biol. 64, 343-369

24. Treisman, R., and Ammerer, G. (1992) Curr. Opin. Genet. Dev. 2, 221-226

25. Buchwalter, G., Gross, C., and Wasylyk, B. (2004) Gene 324, 1-14

26. Shore, P., and Sharrocks, A. D. (1995) Eur. J. Biochem. 229, 1-13 